\title{
Pulse Compression for Weather Radars
}

\author{
Ashok S. Mudukutore, V. Chandrasekar, Member, IEEE, and R. Jeffrey Keeler, Member, IEEE
}

\begin{abstract}
Wideband waveform techniques, such as pulse compression, allow for accurate weather radar measurements in a short data acquisition time. However, for extended targets such as precipitation systems, range sidelobes mask and corrupt observations of weak phenomena occurring near areas of strong echoes. Therefore, sidelobe suppression is extremely important in precisely determining the echo scattering region. A simulation procedure has been developed to accurately describe the signal returns from distributed weather targets, with pulse compression waveform coding. This procedure is unique and improves on earlier work by taking into account the effect of target reshuffling during the pulse propagation time which is especially important for long duration pulses. The simulation procedure is capable of generating time series from various input range profiles of reflectivity, mean velocity, spectrum width, and SNR. Results from the simulation are used to evaluate the performance of phasecoded pulse compression in conjunction with matched and inverse compression filters. The evaluation is based on comparative analysis of the integrated sidelobe level and Doppler sensitivity after the compression process. Pulse compression data from the CSU-CHILL radar is analyzed. The results from simulation and the data analysis show that pulse-compression techniques indeed provide a viable option for faster scanning rates while still retaining good accuracy in the estimates of various parameters that can be measured using a pulsed-Doppler radar. Also, it is established that with suitable sidelobe suppression filters, the range-time sidelobes can be suppressed to levels that are acceptable for operational and research applications.
\end{abstract}

Index Terms-Pulse compression, weather radar.

\section{INTRODUCTION}

$\mathbf{P}$ ULSE compression techniques allow for the transmission of a low peak-power, long-duration coded pulse and attain the fine range resolution and improved detection performance of a short duration, high peak-power pulse system. This is accomplished by widening the bandwidth of the transmitted pulse by coding it in either phase or frequency, which yields a finer range resolution $(\Delta R=c /(2 B))$ than can be achieved with a conventional radar system using an uncoded pulse. The received echo waveform is processed using some variant of a filter matched to the transmit coding scheme which compresses

Manuscript received July 21, 1996; revised January 29, 1997. This work was supported by the U.S. Air Force Office of Scientific Research Grant F4962095-1-0133 and National Science Foundation Grant ATM-9413453. The work of R. J. Keeler was supported by the FAA Terminal Area Surveillance Systems program.

A. S. Mudukutore is with the Research Triangle Institute, Hampton, VA 23666 USA (e-mail: asm@rti.org).

V. Chandrasekar is with the Department of Electrical Engineering, Colorado State University, Fort Collins, CO 80523 USA.

R. J. Keeler is with the Remote Sensing Facility, National Center for Atmospheric Research, Boulder, CO 80307 USA.

Publisher Item Identifier S 0196-2892(98)00035-7. the long pulse to a duration $1 / B$, where $B$ is the bandwidth of the transmit waveform.

The driving force behind exploring pulse compression for military applications is the necessity for greater pulse energy and increased range resolution. The same reason makes it suitable for a number of meteorological applications where high peak powers are difficult or expensive to obtain (e.g., for millimeter-wave systems). Weather radars with high peak power transmitters, however, have adequate sensitivity and range resolution required for many applications. Using pulse compression with weather radars allows them to rapidly scan three-dimensional (3-D) precipitation patterns and trace their evolution with time. This is accomplished as follows. Antenna beams for weather radars are typically circular in crosssection with half-power beamwidths of 0.25 to $3.0^{\circ}$ and pulse lengths on the order of $100 \mathrm{~m}$. Therefore, the pulse volume is "pancake" shaped for ranges beyond 10-20 km, with the cross-range width larger compared to the down-range depth. Thus, it appears that using pulse compression to improve the down-range resolution would have little to offer. However, averaging the fine-scale down-range measurements allows the $d$ well time to be reduced and provides the same or larger number of independent samples, thereby improving accuracy in measurements of the radar received signals.

\section{A. Background}

Pulse compression techniques have been well established for applications in military and aviation systems where the backscattering medium consists of hard targets. Several techniques have been proposed and studied since the early 1950's and are reviewed in a number of textbooks [1], [2]. Use of pulse compression for extended precipitation targets was not investigated until the early 1970's. Fetter [3] demonstrated the use of a 7-bit Barker phase-coded transmit pulse and a matched-filter receiver, implemented on the coherent FPS-18 radar at McGill University. Gray and Farley [4] investigated the use of binary phase-coded pulse compression for incoherent scatter observations. Keeler and Passarelli [5] have traced the evolution of pulse compression techniques in the weather radar community.

\section{B. Description}

Fig. 1 illustrates the basic concept of pulse compression processing in a weather radar system. The signal from the waveform modulator is used to code the radio frequency (RF) signal. Fig. 1 shows a transmit waveform modulated by a 5bit phase code. The received signal is fed through a pulse compression filter, which frequently consists of a matched 


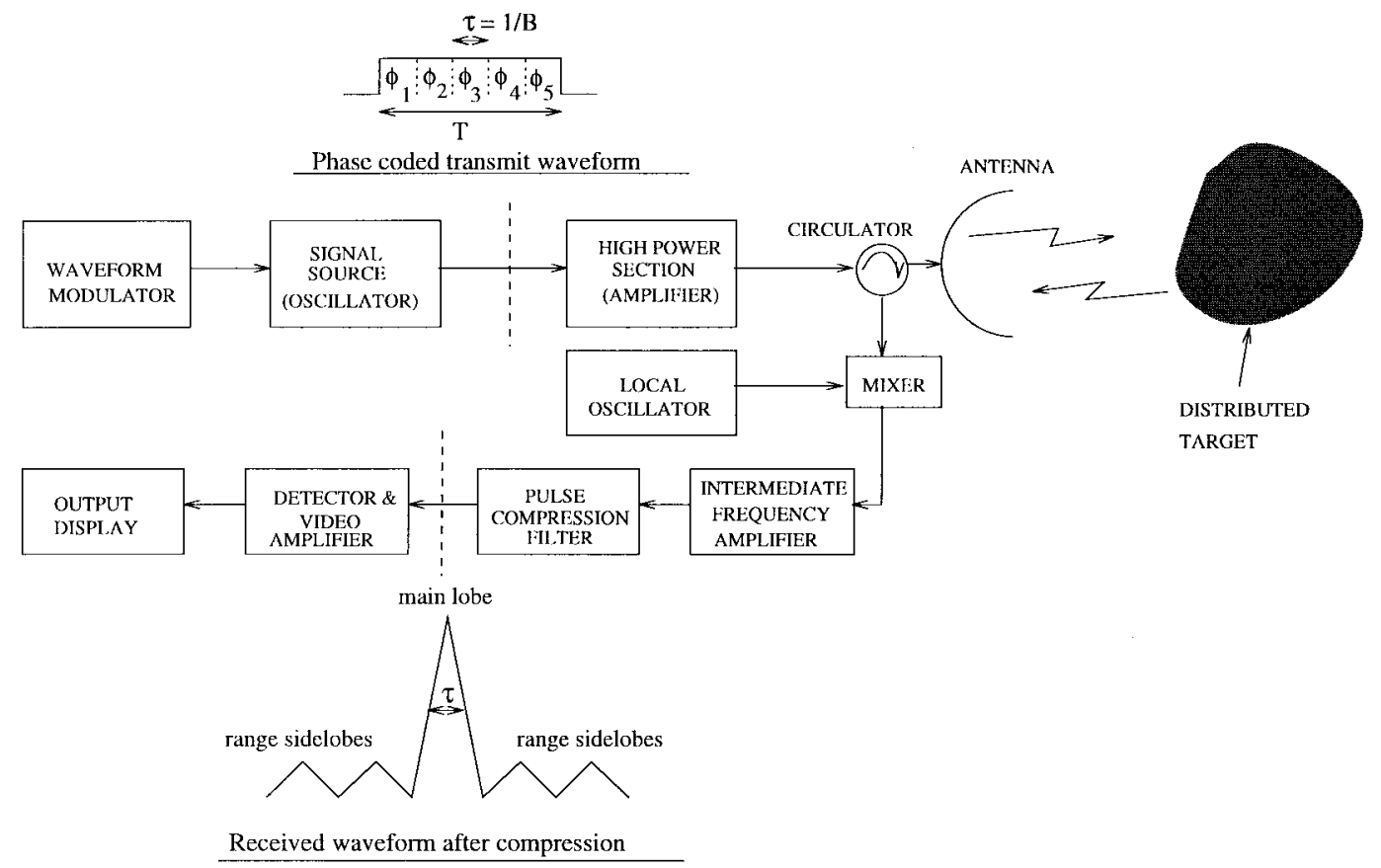

Fig. 1. Block diagram of pulse compression radar.

filter section followed by a sidelobe suppression filter. The peak power of the compressed pulse is increased by the compression ratio $(\mathrm{CR})$ defined as

$$
\mathrm{CR}=B . T=\frac{T}{\tau}
$$

where $T$ is the transmitted pulse length, $B$ is the bandwidth of the transmitted waveform, and $\tau$ is the effective (compressed) pulse length $(\tau=1 / B)$ of the system. This ratio, alternatively known as time-bandwidth product, is a measure of the degree to which the pulse is compressed. For pulse compression waveforms, the compression ratio is greater than 1. Typical values range from 5 to as large as $10^{5}$.

\section{Range Sidelobes and Weighting}

A major drawback to the application of pulse compression is the presence of range sidelobes which tend to smear the returns in range. Suppression of range sidelobes is critical, especially in applications for weather radars where the observed targets are distributed in nature and often have strong and steep gradients in reflectivity. Sidelobe suppression, in general, is achieved by tapering the matched filter response by weighting the transmitted waveform, the matched filter, or both in either frequency or amplitude. The weighting is usually applied to the matched filter which causes a loss of SNR due to the mismatched section. The following measures are often used to quantify the performance of range sidelobe suppression techniques.

- Peak sidelobe level (PSL) is defined as

$$
\text { PSL }=10 \log \frac{\text { peak sidelobe power }}{\text { total mainlobe power }} \text {. }
$$

- Integrated sidelobe level (ISL), a measure of the energy distributed in the sidelobes, is defined as

$$
\text { ISL }=10 \log \frac{\text { power integrated over sidelobes }}{\text { total mainlobe power }} .
$$

- Loss in processing gain (LPG), a measure of loss in SNR due to mismatched as opposed to matched filtering, is defined as

$$
\mathrm{LPG}=10 \log \frac{B . T}{\text { total mainlobe power }} .
$$

\section{Implementation}

The choice of a pulse compression system is dependent on the type of waveform selected and the method of generation and processing. Frequency-modulated pulse compression techniques involve sweeping the carrier frequency of the transmit waveform in a linear or nonlinear fashion. These techniques have been investigated [6], [7] and are known to yield good sidelobe performance. In this paper, however, we consider only phase-coded waveforms because of the ease in implementation for a high-powered, ground-based weather radar system.

Phase-coded pulse compression involves transmitting a long pulse of duration $T$ consisting of $N$ subpulses, each of width $\tau=T / N$. The phase of each subpulse is chosen to be one of two possible values (0 or $\pi \mathrm{rad}$ ) for biphase coding or one of several values for polyphase coding. Various codes with known sidelobe properties have been studied [8]. Important among them are Barker codes, combined Barker codes, pseudorandom codes, etc. Barker codes are the biphase codes having the property that after passage through a matched filter, the resulting sequence has sidelobes of unit magnitude $(\mathrm{PSL}=1 / N)$. Barker codes have the attractive property that 


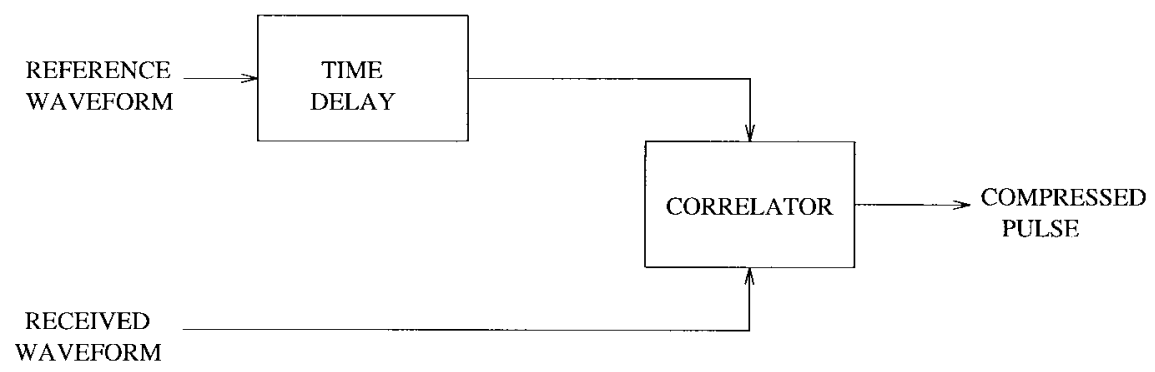

(a)

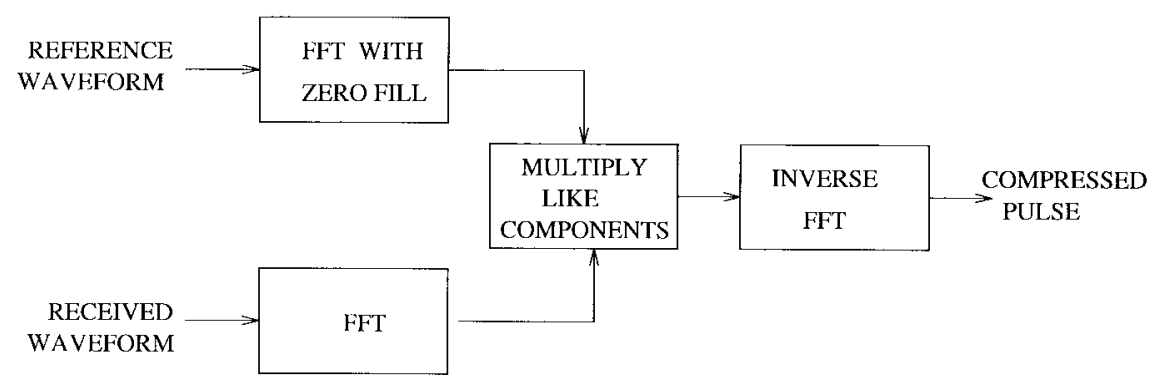

(b)

Fig. 2. Matched filter implementation: (a) correlation processor and (b) FFT processor.

their sidelobe structures contain the minimum energy that is theoretically possible and this energy is uniformly distributed among the sidelobes.

\section{E. Related Issues}

This section outlines the concepts of matched filtering and ambiguity functions which are of fundamental importance to the understanding of pulse compression.

1) Matched Filter: For a received waveform $s(t)$, it can be shown that the frequency-response function of a linear, time-invariant filter which maximizes the output peak-signal to mean-noise ratio for a fixed input SNR is [9]

$$
H(f)=S^{*}(f)
$$

where $S(f)$ is the Fourier transform of $s(t)$ and * superscript denotes the complex conjugation. The filter whose frequency response is given by (5) is called the matched filter. In obtaining (5), it is assumed that the noise accompanying the signal is stationary and has a uniform spectrum (white noise). The impulse response of the matched filter, therefore, is given by

$$
h(t)=s^{*}(-t) .
$$

Fig. 2 shows the time- and frequency-domain implementations of the matched filter. Both implementations are widely used and the choice between them depends on the waveform and the domain (time or Doppler) over which the receiver must process signals.

2) Radar Ambiguity Function: The study of radar waveforms would be quite straightforward if the scatterers in the radar resolution volume were stationary. However, when there is a significant Doppler shift, the reflections from even a point target are no longer replicas of the transmit waveform.
The radar ambiguity function proposed by Woodward [10] quantitatively describes the interference to a reference target caused by targets which are range- and Doppler-shifted with respect to the reference target. For a range delay $T_{R}$ and Doppler frequency $f_{d}$, the cross ambiguity function is defined as

$$
\Psi_{u v}\left(T_{R}, f_{d}\right)=\left|\int_{-\infty}^{\infty} u(t) v^{*}\left(t+T_{R}\right) \exp \left(j 2 \pi f_{d} t\right) d t\right|^{2}
$$

where $u(t)$ is the transmit waveform and $v(t)$ is the impulse response of the filter.

\section{Simulation of Dispersed Radar Pulse Weather Echo}

This section addresses the problem of accurately simulating radar echoes from coded wideband waveforms in the context of distributed, time-varying targets. If the scatterers in the radar resolution volume were stationary or move with identical velocities, the dispersed pulse echo could be achieved by passing the complex signal from the simple pulse echo through a filter whose impulse response is the desired dispersed pulse transmission. This simple model cannot be applied to a distributed weather medium consisting of scatterers moving with different velocities. The Doppler shifts resulting from phase fluctuations during the course of passage of the dispersed pulse are responsible for the Doppler sensitivity of various sidelobe suppression techniques. In addition, echoes from distributed weather targets have pulse-to-pulse fluctuation controlled by the velocity distribution of the scatterers. In order to be able to see the fluctuation that takes place during the passage of the dispersed pulse, one needs to simulate the signal characteristics at a time-scale corresponding to the propagation time over a resolvable range bin, rather than observations separated by the 
pulse repetition period. A new simulation technique is developed here to accurately describe the statistical characteristics (the joint-distribution) of radar returns of a coded waveform from distributed targets, for both single and dual polarization operation. The simulation procedure accounts for the effect of reshuffling of scatterers during the pulse propagation time. This is especially important while considering long duration pulses $(>10 \mu \mathrm{s})$.

\section{A. Radar Signals from Weather Targets}

The inphase $(I)$ and quadrature $(Q)$ components of the complex echo signal from a weather target have a Gaussian distribution with zero mean [11]. Therefore, their probability density functions are given by

$$
\begin{aligned}
& p(I)=\frac{1}{\sqrt{2 \pi} \sigma} e^{-I^{2} / 2 \sigma^{2}}, \quad-\infty<I<+\infty \\
& p(Q)=\frac{1}{\sqrt{2 \pi} \sigma} e^{-Q^{2} / 2 \sigma^{2}}, \quad-\infty<Q<+\infty
\end{aligned}
$$

where $\sigma^{2}$ is the variance of the $I$ and $Q$ samples (also the mean square value because $I$ and $Q$ are zero-mean). In addition to being Gaussian random variables, the $I$ and $Q$ components are independent of each other [12]. The power spectrum of weather signals, also referred to as the Doppler spectrum in velocity space, is a power-weighted distribution of radial velocities of all scatterers that lie in a resolution volume. The first three moments of the power spectrum are directly related to the reflectivity, mean radial velocity, and velocity spectrum width, respectively. The Doppler spectrum can be approximated to be of Gaussian shape sitting on a white noise pedestal, i.e.,

$$
S(v)=\frac{\mathrm{S}}{\sqrt{2 \pi} \sigma_{v}} e^{-(v-\bar{v})^{2} / 2 \sigma_{v}^{2}}+\frac{2 N T_{s}}{\lambda}
$$

where $S$ is the mean signal power, $\bar{v}$ is the mean velocity, $\sigma_{v}$ is the spectrum-width, $N$ is the mean white noise power, $\lambda$ is the wavelength, and $T_{s}$ is the time spacing between samples.

\section{B. Simulation Technique Description}

The simulation procedure to obtain a dispersed radar echo is a two-step process.

1) Simple Pulse Echo Simulation: The time series from a simple pulse can be simulated using the simulation algorithm for multivariate signals described by Chandrasekar et al. [13]. This procedure constructs the Doppler spectrum at each range bin based on the distribution properties of the signal and range profiles of radar reflectivity $(Z)$, mean velocity $(\bar{v})$, velocity spectrum width $\left(\sigma_{v}\right)$, and SNR. The complex time series is then obtained via an inverse DFT. For dual-polarization operation, in addition to the parameters listed above, the differential reflectivity $\left(Z_{\mathrm{DR}}\right)$ and correlation between horizontally $(\mathrm{H})$ and vertically $(\mathrm{V})$ polarized returns $\left(\rho_{\mathrm{HV}}\right)$ are also specified. Separate time series are then generated at $\mathrm{H}$ and $\mathrm{V}$ polarizations. The time spacing between adjacent samples is chosen to be $T_{s}=1 / B$ ( $B=$ transmit waveform bandwidth). This is in contrast to the procedure used by [13] where $T_{s}=T_{\mathrm{PRT}}$, the pulse repetition time. The finer time-scale is necessary to accurately describe the joint-distribution characteristics of the radar returns as the dispersed pulse propagates through the medium. This procedure yields a two-dimensional (2-D) complex array $S[m, n],\left(m=1, \cdots, n_{\text {bins }} ; n=1, \cdots, n_{\text {tot }}\right)$, where $n_{\text {tot }}$ represents the number of samples at each range bin separated by $T_{s}$, and $n_{\text {bins }}$ is the number of range bins.

2) Evolution of the Dispersed Pulse Echo: The effect of the modulation waveform $\left(p[n], n=1, \cdots, n_{p}\right)$ on the simple pulse time series $S[m, n]$ is incorporated in the new representation of the echo-signal shown below

$$
x_{i}[m, n]=S\left[m,(i-1) n_{d}+n\right] p[n]
$$

where $i$ is the sample-time index (i.e., samples separated by $\left.T_{\mathrm{PRT}}\right)$ and $n_{d}=T_{\mathrm{PRT}} / T_{s}$. The evolution of the dispersed echo from $x_{i}[m, n]$ is shown in Fig. 3 for a 5-bit phase code and explained below. Given that $T_{s}=1 / B=\tau$ ( $\tau$ is the subpulse duration), each subpulse in the transmit pulse defines a range bin. As the pulse at any sample-index $i$ propagates, at the first range-sampling instant, the first subpulse encounters the first range bin $(1,1)$ contributing to the first echo $y[i, 1]$. As the pulse moves on, at the next range-sampling instant, the first subpulse now encounters the second range-bin $(2,1)$ and the second subpulse encounters the first range bin $(1,2)$ and the combination of these two gives yields echo sample $y[i, 2]$. Echoes at other ranges are similarly obtained. The echo construction procedure explained here is similar to that used by Bucci and Urkowitz [14], and is mathematically described as

$$
y[i, j]=\sum_{\forall m+n-1=j} x_{i}[m, n]
$$

\section{RANGE SIDELOBE SUPPRESSION}

Due to the distributed nature of weather targets, the integrated sidelobe level (ISL) provides a good measure of range sidelobe contamination. While evaluating the performance of pulse compression systems, generally speaking, any waveform-filter combination is considered suitable if it yields an ISL comparable to the sidelobe contribution from a typical two-way antenna pattern. In this section, based on the simulation procedure described in Section II, the performance of matched and inverse range sidelobe suppression filters are evaluated. The criteria used in evaluation are integrated sidelobe level (ISL), Doppler sensitivity after the compression process, and how well the estimates of various parameters after pulse compression match up with those obtained from simple pulses.

\section{A. Inverse Filter}

Ackroyd and Ghani [15] discuss an optimal filtering technique for minimizing the ISL of the code response in a least squares sense. This filter, called inverse filter, can be implemented to act directly on the dispersed echo or on the output of the matched filter. By implementing this optimal ISL technique, the PSL and ISL levels can be driven to very low values. For example, for a 13-bit Barker code, a modest filter length of 39 (code-length $\times 3$ ) yields a PSL of $-38 \mathrm{~dB}$ and an ISL of $-30 \mathrm{~dB}$. Application of optimal ISL filtering techniques to combined Barker codes also yields good results [16]. 


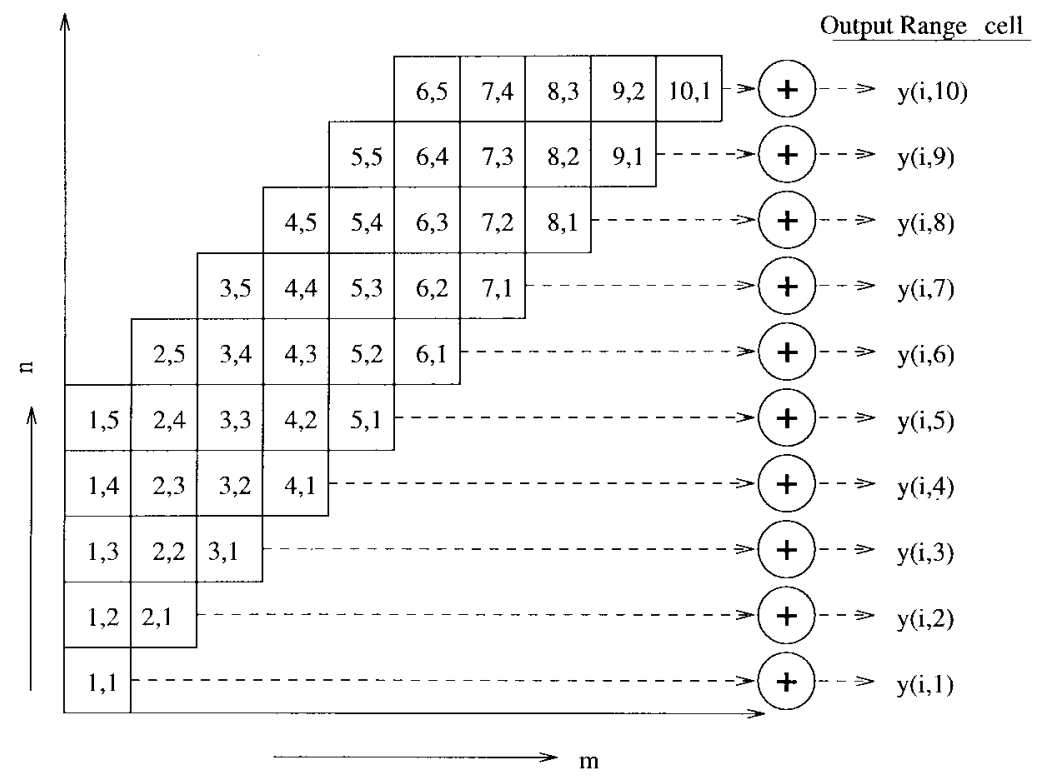

Fig. 3. Evolution of dispersed echo at sample-time index $i$. Each square labeled $(m, n)$ represents sample $x_{i}(m, n)\left(m=1, \cdots, n_{\text {bins }} ; n=1, \cdots, n_{p}\right)$ and the resulting output is shown on the right. In figure, $n_{\text {bins }}=10$ and $n_{p}=5$.

1) Filter Design: We will restrict ourselves to the design of an optimal ISL filter to act directly on the dispersed echosignal in contrast with other schemes which could operate on the signal at the output of the matched filter. Let $\left(x_{k}, k=\right.$ $1, \cdots, N)$ represent the input signal, $\left(w_{k}, k=1, \cdots, M\right)$ represent the weighting sequence of the filter, and $\left(y_{k}, k=\right.$ $1, \cdots, M+N-1)$ represent the sequence at the output of the filter. Let $\left(d_{k}, k=1, \cdots, M+N-1\right)$ be the desired response, defined here to be an impulse function. The filtering criterion is the minimization of the mean-squared-error, defined as

$$
V=E\left[\left(d_{k}-y_{k}\right)^{2}\right] .
$$

The optimum weight-vector $\mathbf{w}_{\mathbf{o}}$, satisfying (13), is obtained by solving the vector-matrix equation

$$
\mathbf{R} \mathbf{w}_{o}=\mathbf{p}
$$

where $\mathbf{R}$ is the auto correlation matrix of $x_{k}$ and $\mathbf{p}$ is the discrete cross-correlation of $x_{k}$ and $d_{k}$ [17]. Equation (14) is the discrete form of the Wiener-Hopf equation.

2) Doppler Tolerant Implementation: The performance of the matched filter as well as the inverse filter degrades in the presence of a Doppler shifted radar return. This is due to the fact that both the matched filter and the inverse sidelobe suppression filter are designed for optimal performance under zero Doppler velocity conditions. A measure of the Doppler sensitivity can be obtained from the Doppler phase shift over the pulse duration given by $\phi_{s}=2 \pi f_{d} T$, where $f_{d}$ is the Doppler frequency shift and $T$ is the duration of the uncompressed pulse. Urkowitz and Bucci [18] outline a Doppler tolerant sidelobe suppression technique which alleviates the sensitivity of the ISL on the target Doppler velocity. The technique suggests passing the received complex signal through a filter-bank to separate out the signal into several Doppler-bins. The signal still contains the Doppler phase shift across the pulse (i.e., along the range samples).
Each Doppler filter output is then multiplied with a complex exponential phase term, corresponding to the Doppler phase obtained from the center frequency of that filter but of opposite polarity, to remove the residual Doppler phase along the range samples. The resulting waveform is then passed through standard sidelobe suppression filters.

\section{B. Evaluation Based On a Point Target}

The evaluation presented here are based on using the following model.

- Modulation waveform: Barker biphase code of length 13 (B-13).

- Wavelength $\lambda=0.1 \mathrm{~m}$.

- Bandwidth $B=1.024 \mathrm{MHz}$.

- Transmit pulse-length $T \simeq 12.7 \mu \mathrm{s}$.

Fig. 4 shows the ambiguity function of the B-13 waveform using (a) matched filter (MF), (b) inverse filter of length 65 (IF $\times \mathbf{5}$ ), and (c) IF $\times \mathbf{5}-\mathrm{DT}$, the Doppler tolerant implementation of IF $\times \mathbf{5}$. The range of the Doppler velocities shown in the Fig. 4 is $[0,50] \mathrm{m} / \mathrm{s}$ which is typical for weather targets. Note that this corresponds to twice the Nyquist velocity $\left(V_{\text {nyq }}=\lambda /\left(4 T_{\mathrm{PRT}}\right)=25 \mathrm{~m} / \mathrm{s}\right)$ for a coherent S-band radar system operating at a pulse repetition time $T_{\mathrm{PRT}}$ of $1 \mathrm{~ms}$. Due to symmetry about the zero Doppler velocity axis $(\mathrm{vel}=0)$, the ambiguity function over the Doppler velocity interval $[0,-50]$ $\mathrm{m} / \mathrm{s}$ would be identical. For the matched filter output, the PSL is constant at $-22.27 \mathrm{~dB}$ and the ISL is $-11.48 \mathrm{~dB}$ over the entire Doppler velocity range shown in the figure.

The output of the inverse filter shows a much lowered sidelobe level $(\mathrm{PSL} \approx-60 \mathrm{~dB}$, ISL $\approx-50 \mathrm{~dB}$ at zero velocity). However, note the increased sensitivity of the output of the inverse filter with Doppler velocity. This sensitivity is reduced using the Doppler tolerant processing scheme shown in panel (c). The Doppler tolerant scheme uses time series samples spaced $T_{\mathrm{PRT}}$ apart as input to a Doppler filter bank. 


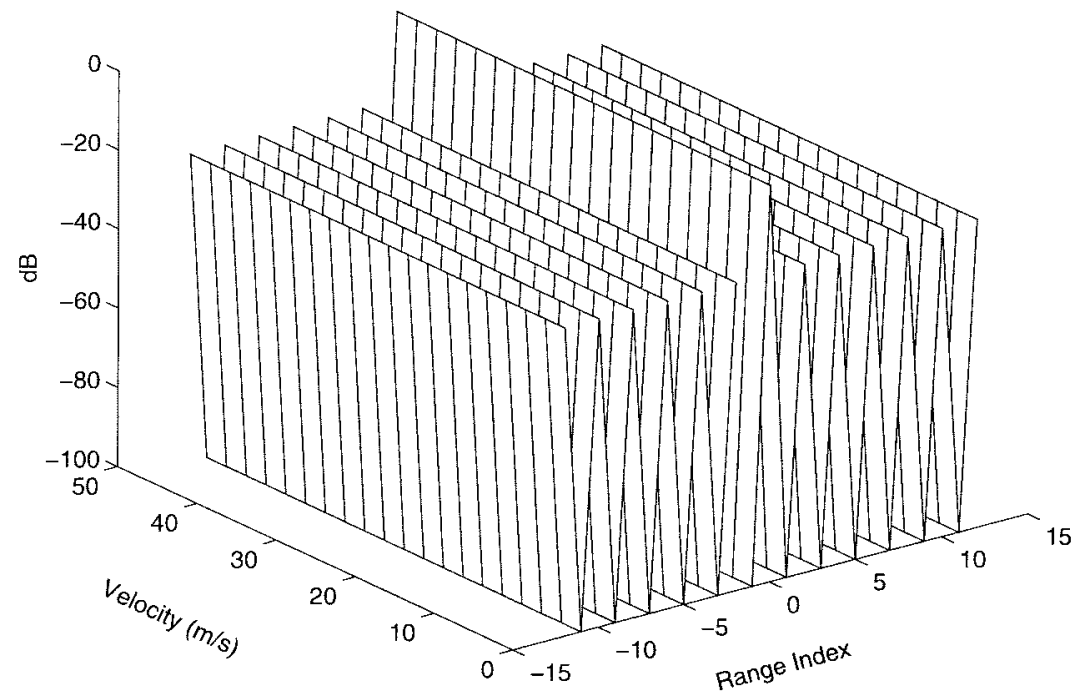

(a)

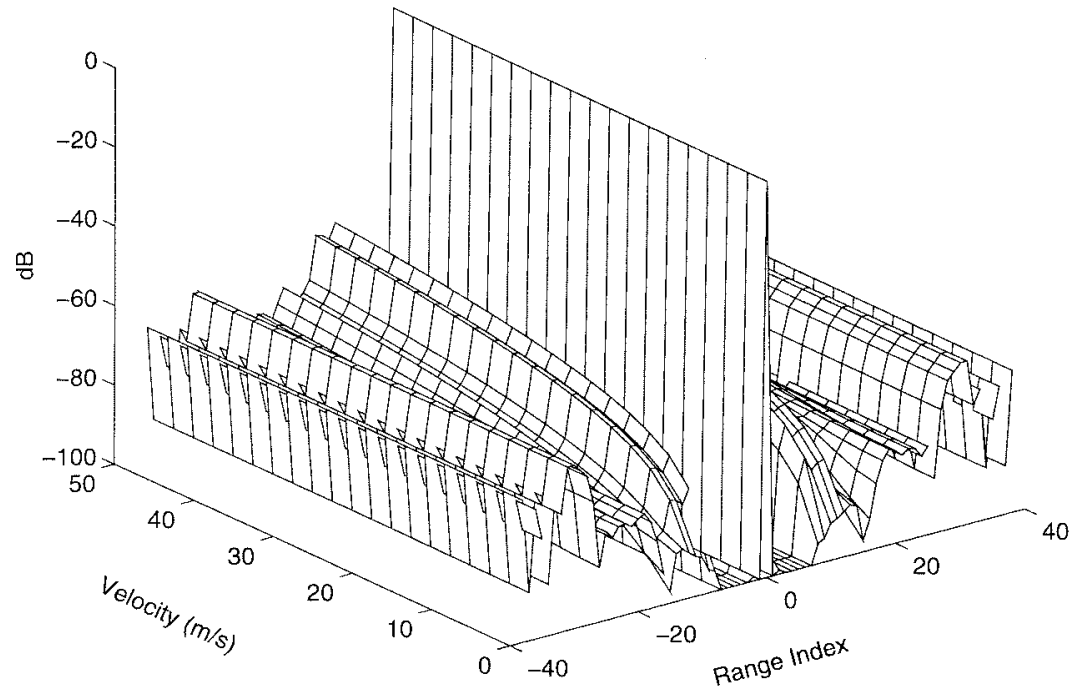

(b)

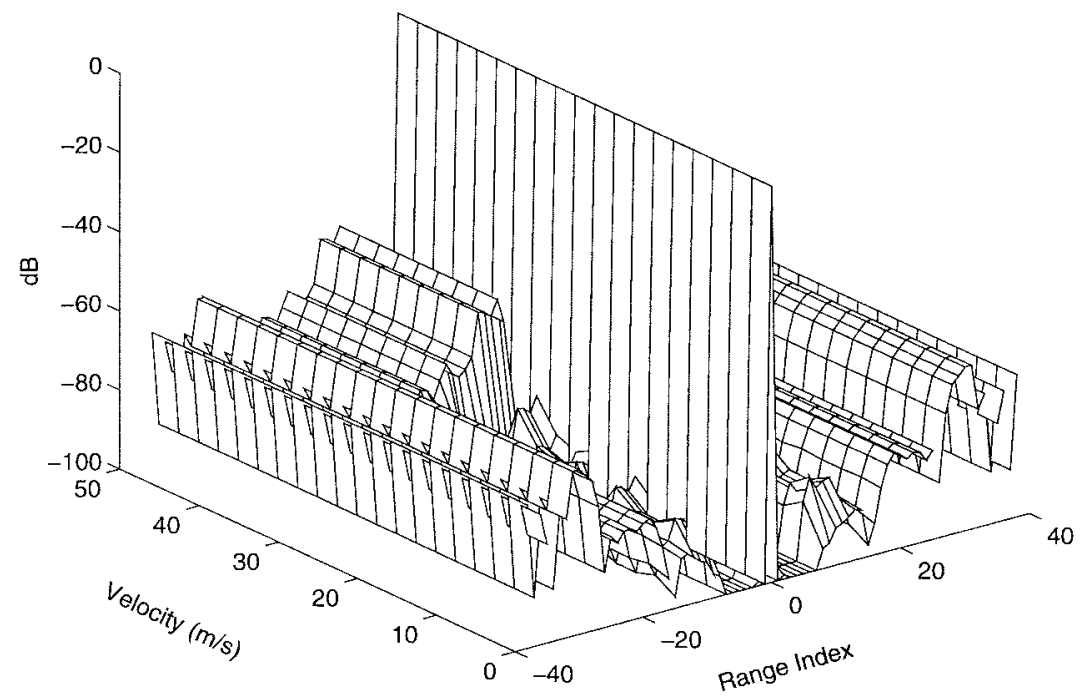

(c)

Fig. 4. Ambiguity function based on point target analysis for the B-13 waveform. The compression filters used are (a) MF (range index from -12 to 12), (b) $\mathbf{I F} \times \mathbf{5}$ (range index from -38 to 38 ), and (c) $\mathbf{I F} \times \mathbf{5}$-DT (range index from -38 to 38 . 

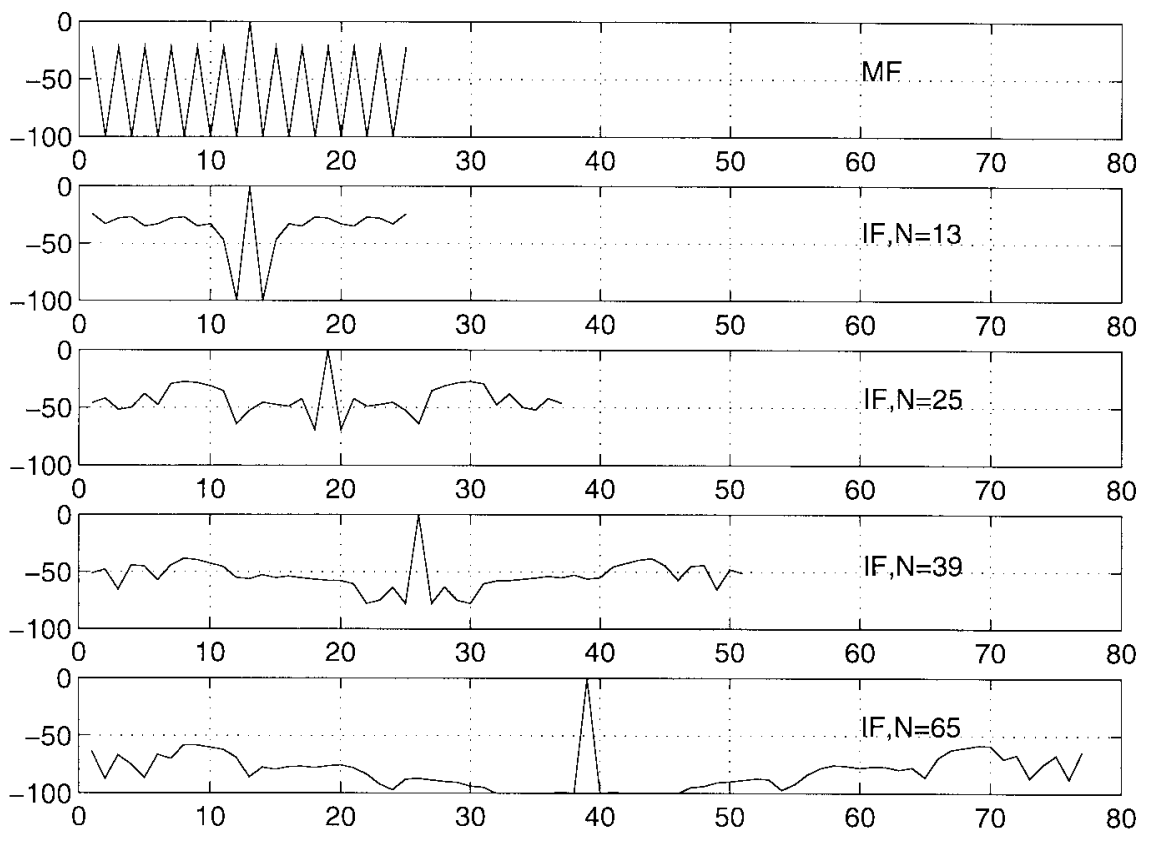

Fig. 5. Compression filter response to B-13 waveform for matched filter $(\mathbf{M F}>)$ and inverse filters of length $13(\mathbf{I F} \times \mathbf{1}), 25(\mathbf{I F} \times \mathbf{2}), 39(\mathbf{I F} \times \mathbf{3})$, and $65(\mathbf{I F} \times \mathbf{5})$ at zero Doppler velocity.

Therefore, the frequency domain representation of the time signal at the output of the filter bank lies in the fundamental Nyquist interval $\left(-1 / 2 T_{\mathrm{PRT}}, 1 / 2 T_{\mathrm{PRT}}\right)$ corresponding to the velocity interval ( $-V$ nyq, $V$ nyq). Thus, for radial velocities outside the Nyquist interval, the multiplication of the complex exponential phase term at the output of each filter bank corresponding to the center frequency results in incorrect compensation of the Doppler phase along the range samples. This effect is evident in Fig. 4(c) for velocities greater than $25 \mathrm{~m} / \mathrm{s}$, where we see that the output of the Doppler tolerant scheme yields higher range sidelobes than the inverse filter without Doppler tolerance.

Fig. 5 shows the output of the matched filter and inverse filters of different lengths for a stationary point target. The MF consistently yields higher sidelobes. The inverse filter sidelobes are much lower and located away from the main response with increasing filter length. For the B-13 waveform under consideration, the loss in processing gain due to mismatch (LPG) is less than $0.5 \mathrm{~dB}$ for all the inverse filters under consideration (i.e., $\mathbf{I F} \times \mathbf{1}$ through $\mathbf{I F} \times \mathbf{7}$ ). Fig. 6(a) shows the ISL versus Doppler velocity curves for the B-13 waveform in the Doppler velocity interval $[0,50] \mathrm{m} / \mathrm{s}$. The different curves correspond to outputs of matched filter (labeled MF), inverse filters of length 13,65 , and 91 (labeled $\mathbf{I F} \times \mathbf{1}, \mathbf{I F} \times \mathbf{5}$ and $\mathbf{I F} \times \mathbf{7}$ ), and the Doppler tolerant implementation of $\mathbf{I F} \times \mathbf{5}$ and $\mathbf{I F} \times \mathbf{7}$ (labeled $\mathbf{I F} \times \mathbf{5}$-DT and $\mathbf{I F} \times \mathbf{7}$-DT, respectively). The MF yields the highest ISL. The ISL for the inverse filters improve with increasing filter length. Also, the sensitivity of the ISL with Doppler shifts increases with filter length (see curves for $\mathbf{I F} \times \mathbf{1}, \mathbf{I F} \times \mathbf{5}$ and $\mathbf{I F} \times \mathbf{7}$ ). The Doppler tolerant implementation does a good job of maintaining low ISL's for all Doppler shifts within the Nyquist interval (see $\mathbf{I F} \times \mathbf{5}$-DT and $\mathbf{I F} \times \mathbf{7}$-DT). Note the sudden increase in ISL at $25 \mathrm{~m} / \mathrm{s}$ due to incorrect phase compensation at the Doppler filter-bank output. Fig. 6(b) plots the ISL versus filter length for the B-13 waveform using inverse compression filters at zero Doppler. Note that the higher order filters (lengths $>90$ ) yield ISL levels of less than $-70 \mathrm{~dB}$.

\section{Evaluation Based On Time-Varying, Distributed Targets}

We demonstrate the application of the matched and inverse filters by analyzing four profiles generated by the new simulation procedure described in Section II. A more detailed discussion on the results from the first three profiles described below can be found in Mudukutore et al. [19], [20].

1) Evaluation of ISL Using a Reflectivity Notch: Consider an input profile consisting of a constant reflectivity level at all ranges except for a deep reflectivity notch at one of the range bins. For time-varying distributed targets, a measure of the ISL can be obtained by computing the ratio of power of the compressed signal at the notch to the power at any other range. However, this procedure does not yield the ISL for hard targets due to the fact that for a fluctuating target, the contributions to the total power at a given range bin from adjacent range bins add up incoherently as opposed to coherent addition for a hard target. The ratio $\mathrm{P}_{\text {notch }} / \mathrm{P}_{\text {other }}$ then reduces to (3) for the fluctuating target. Fig. 7 shows the range profile of the returned power at the output of various compression filters for a input profile with a $100 \mathrm{~dB}$ notch at the center range bin from (a) hard target and (b) random distributed target with $\bar{v}=0, \sigma_{v}=2.5 \mathrm{~m} / \mathrm{s}$ and SNR = $80 \mathrm{~dB}$, for all ranges. The ISL values for the time-varying distributed targets computed in this fashion are consistent with those shown in Section III-B. Fig. 7 specifically brings out the power of our simulation algorithm showing the distinction 


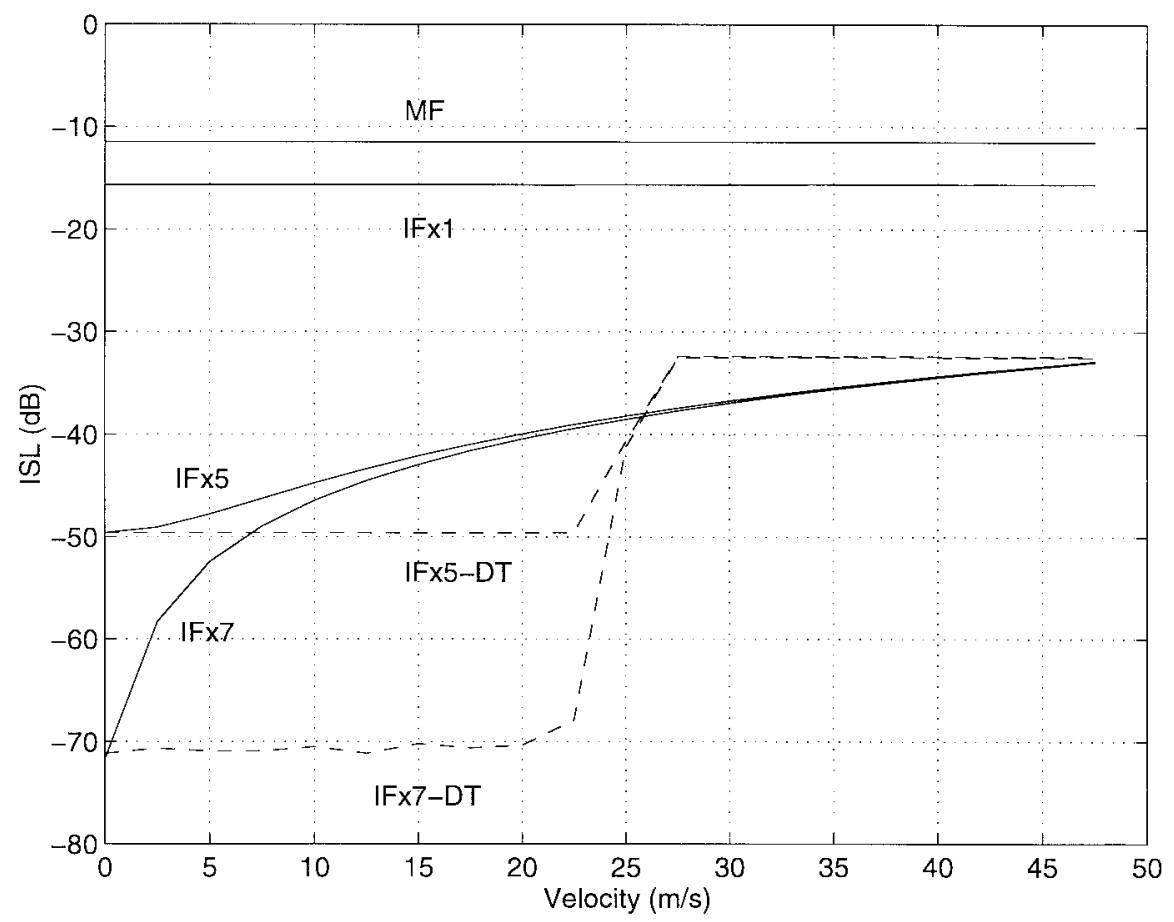

(a)

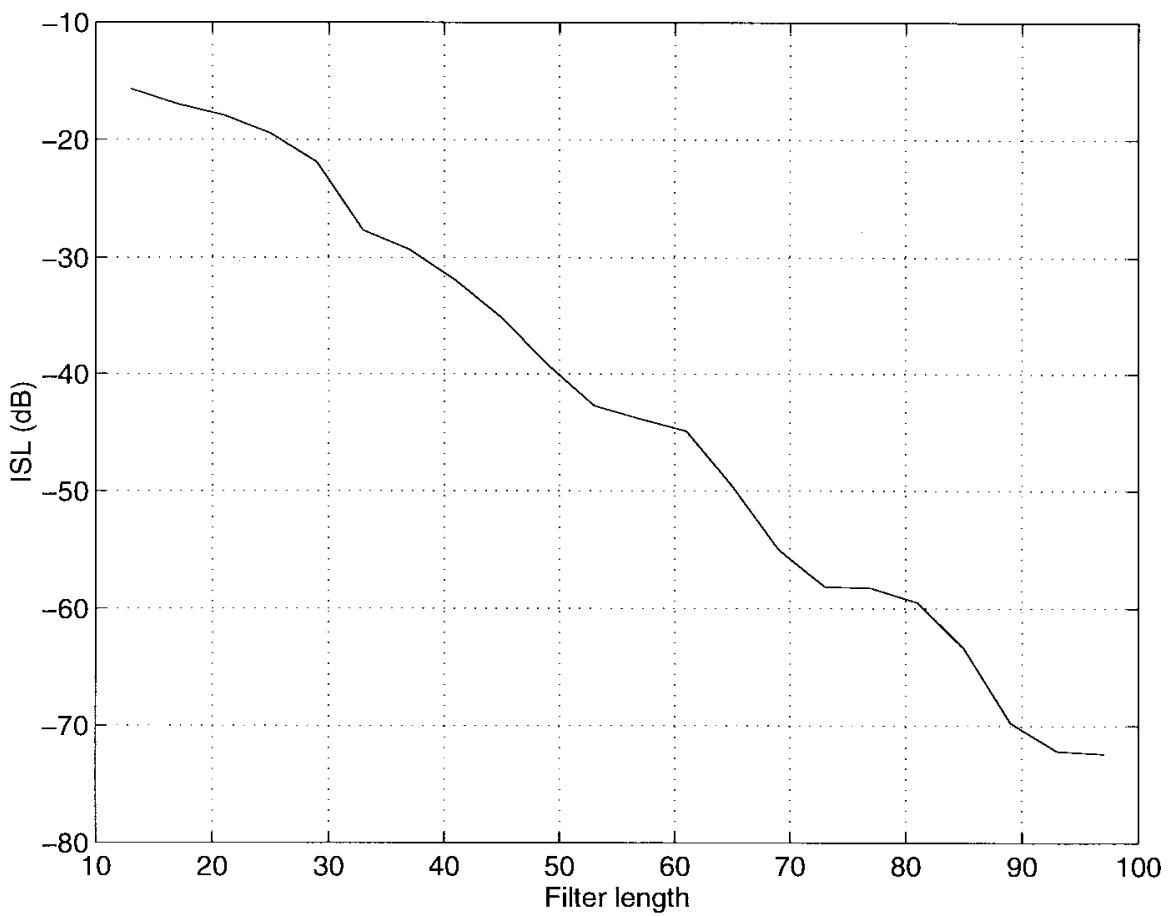

(b)

Fig. 6. (a) ISL versus Doppler velocity for a point target using the B-13 waveform. (b) ISL versus filter length for the B-13 waveform using inverse compression filters for zero Doppler velocity.

between hard target responses and time-varying, distributed target responses.

2) Evaluation of ISL Using a Reflectivity Spike: The input reflectivity profile consists of a $100 \mathrm{~dB}$ spike at the center range bin. The range sidelobes due to the echo spill-over from the spike dominate the power levels in the adjacent range bins. Integrating the power levels in the sidelobes yields a measure of the ISL, which is plotted in Fig. 8 as a function of mean Doppler velocity for a time-varying, distributed target with (a) Doppler spectrum width $\sigma_{v}=1 \mathrm{~m} / \mathrm{s}, \mathrm{SNR}=50$ $\mathrm{dB}$; (b) $\sigma_{v}=1 \mathrm{~m} / \mathrm{s}, \mathrm{SNR}=80 \mathrm{~dB}$. It can be seen from Fig. 8 that the responses of the various filters to the fluctuating 


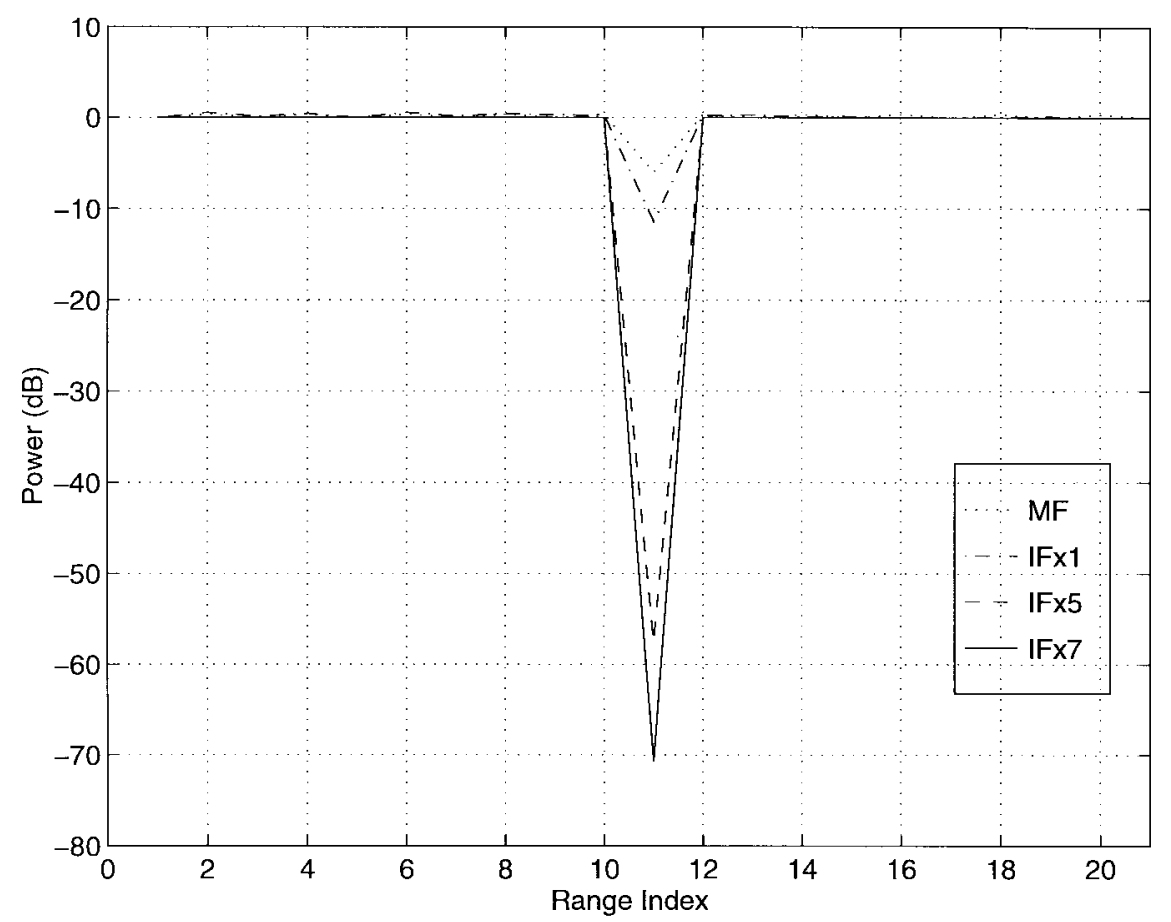

(a)

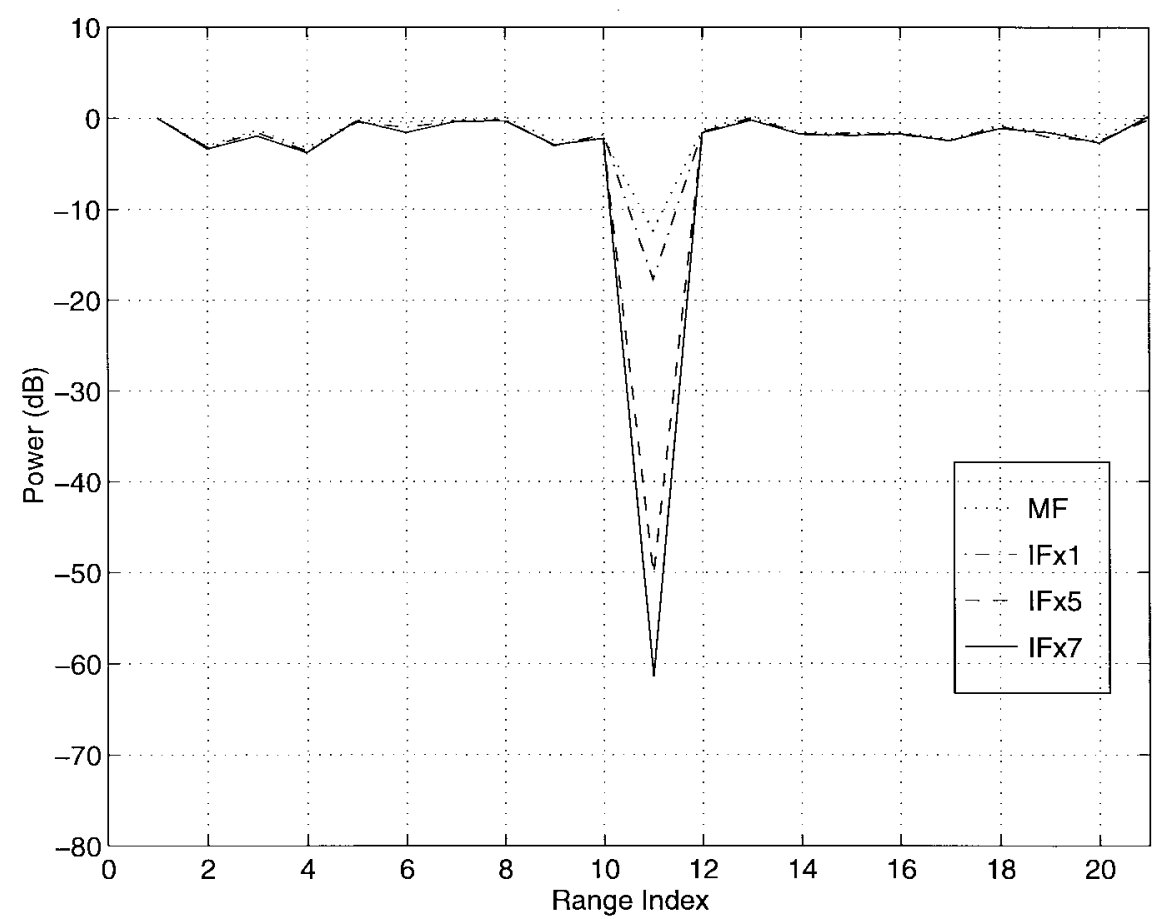

(b)

Fig. 7. Range profiles of returned power at output of various compression filters for input notch profile (100 dB notch) using (a) hard target and (b) time-varying distributed target.

targets is similar to the hard target response shown in Fig. 6(a). However, there are a couple of interesting differences from the hard target response, especially at longer filter lengths.

a) Effect of spectrum width: As explained before, the ISL for IF $\times \mathbf{5}$-DT and IF $\times \mathbf{7}$-DT increases steeply at the Nyquist interval, for hard target responses. However, for fluctuating responses, the performance of these Doppler tolerant implementations degrades as the velocities approach the extremities of the unambiguous Nyquist interval. This is due to the fact that at these higher velocities, the aliased portions of the Doppler spectrum wrap around the Nyquistinterval, resulting in incorrect Doppler phase compensation at the output of the Doppler filter-bank. The situation gets worse with larger spectrum widths as can be seen from Fig. 9. 


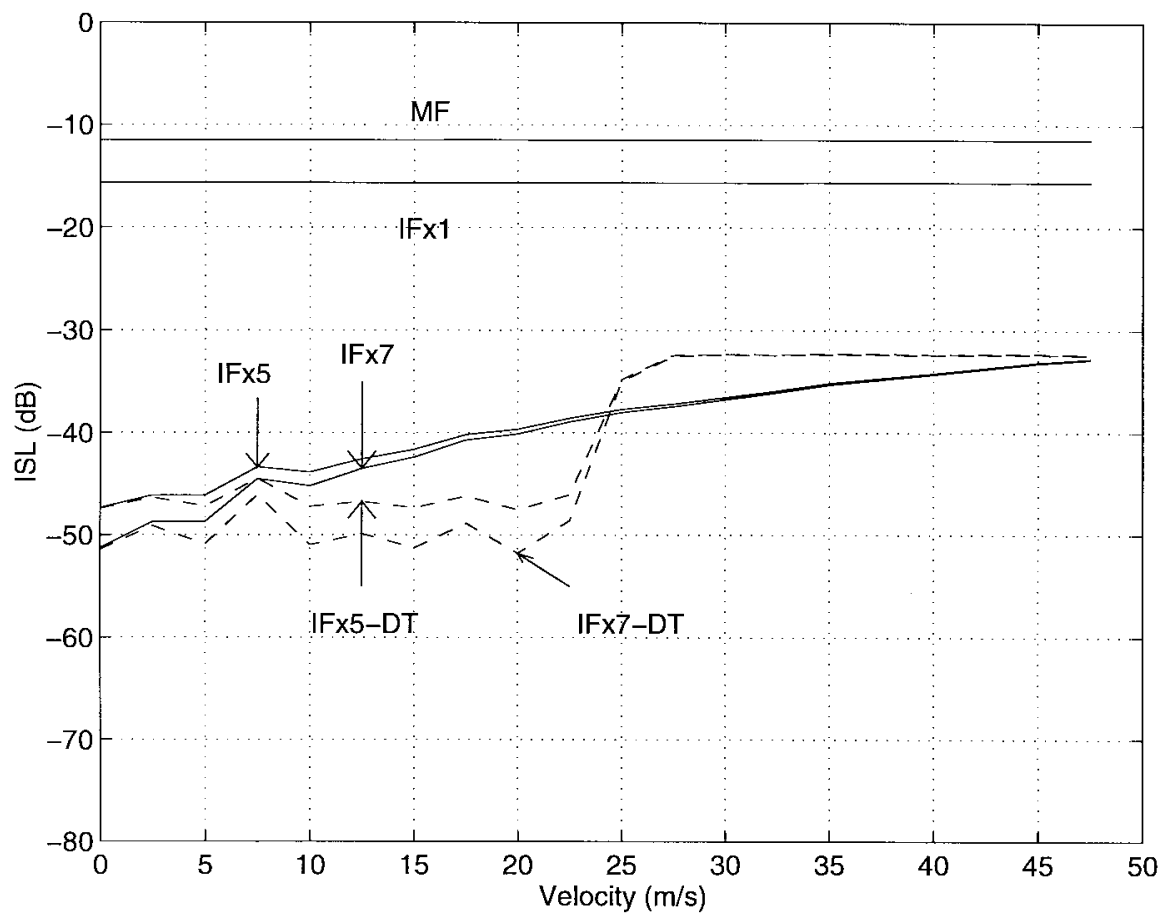

(a)

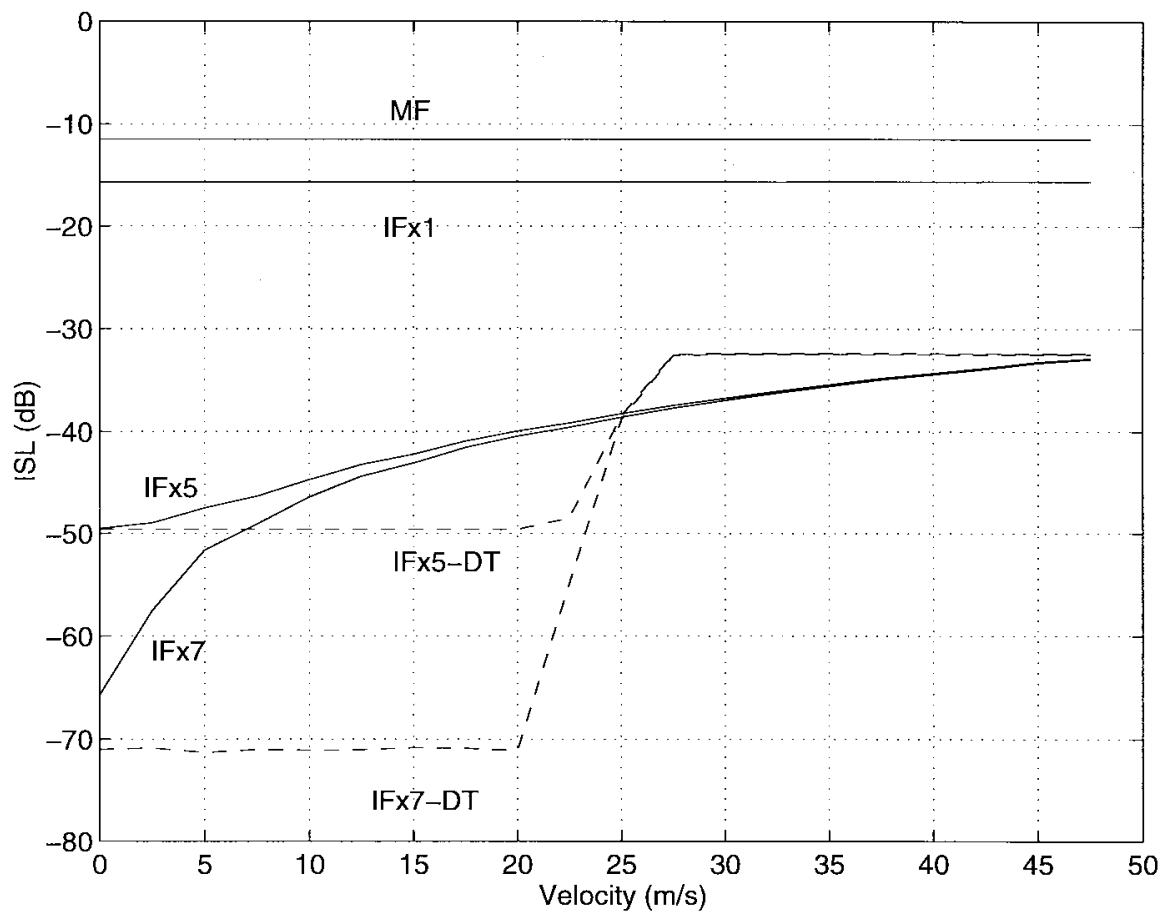

(b)

Fig. 8. ISL versus Doppler velocity from a time-varying, reflectivity spike with (a) $\sigma_{v}=1 \mathrm{~m} / \mathrm{s}, \mathrm{SNR}=50 \mathrm{~dB}$ and (b) $\sigma_{v}=1 \mathrm{~m} / \mathrm{s}, \mathrm{SNR}=80 \mathrm{~dB}$.

b) Effect of SNR: The outputs $\mathbf{I F} \times \mathbf{7}$ and $\mathbf{I F} \times \mathbf{7}-\mathbf{D T}$ in Fig. $8(\mathrm{a})(\mathrm{SNR}=50 \mathrm{~dB})$ do not match with those for the hard target. However, the same outputs match up well with the hard target response in Fig. 8(b) $(\mathrm{SNR}=80 \mathrm{~dB})$. This suggests that the minimum ISL that can be obtained for any inverse filter is limited by the SNR. This can be explained by the fact that as the coded dispersed waveform propagates through the distributed medium, the fluctuations of the signal occurring at a time-scale corresponding to the range sampling time (inverse bandwidth) are small and do not decorrelate the signal significantly from one range sampling instant to the next. However, the effect of the fluctuating noise is to add a random phase component to the underlying phase modulation of the transmit waveform, thereby degrading the performance 


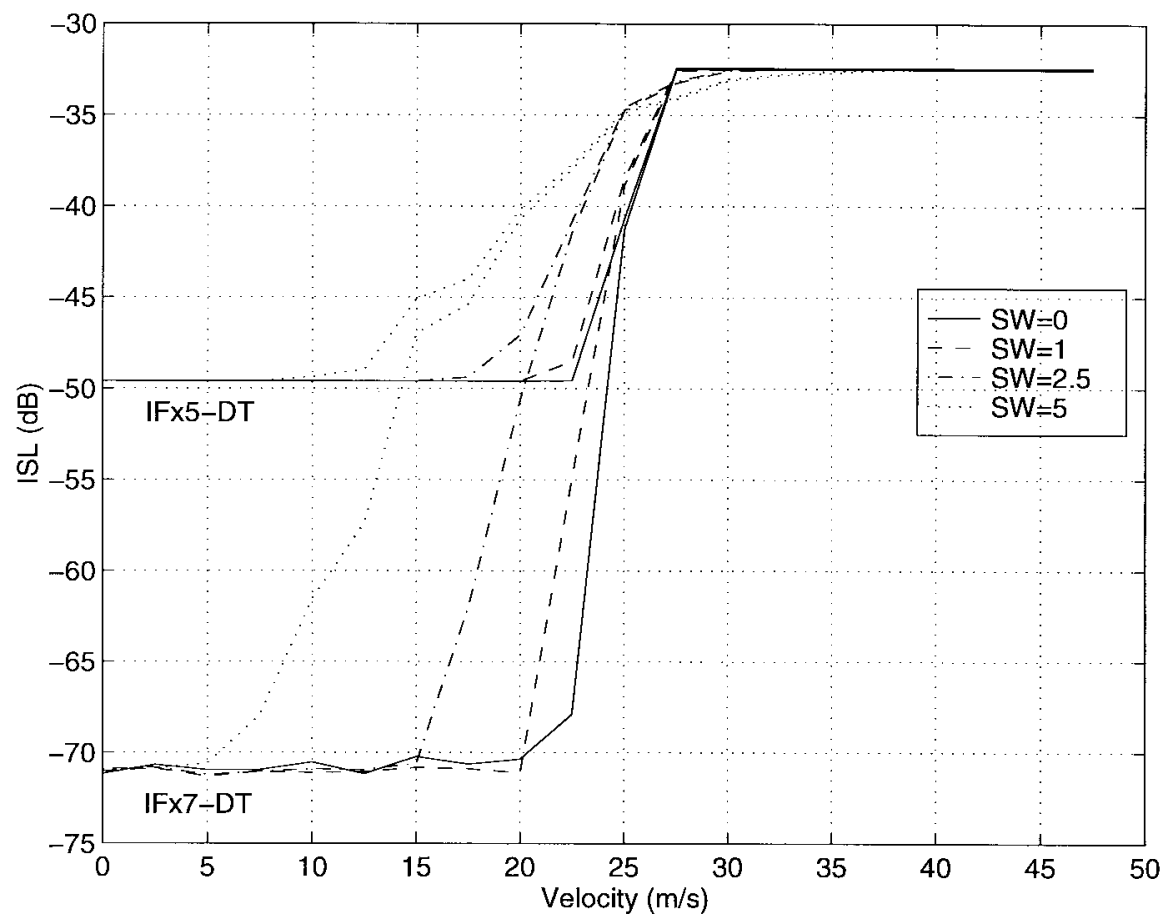

Fig. 9. ISL versus Doppler velocity for $\mathbf{I F} \times \mathbf{5}-\mathbf{D T}$ and $\mathbf{I F} \times \mathbf{7}$-DT at various spectrum widths.

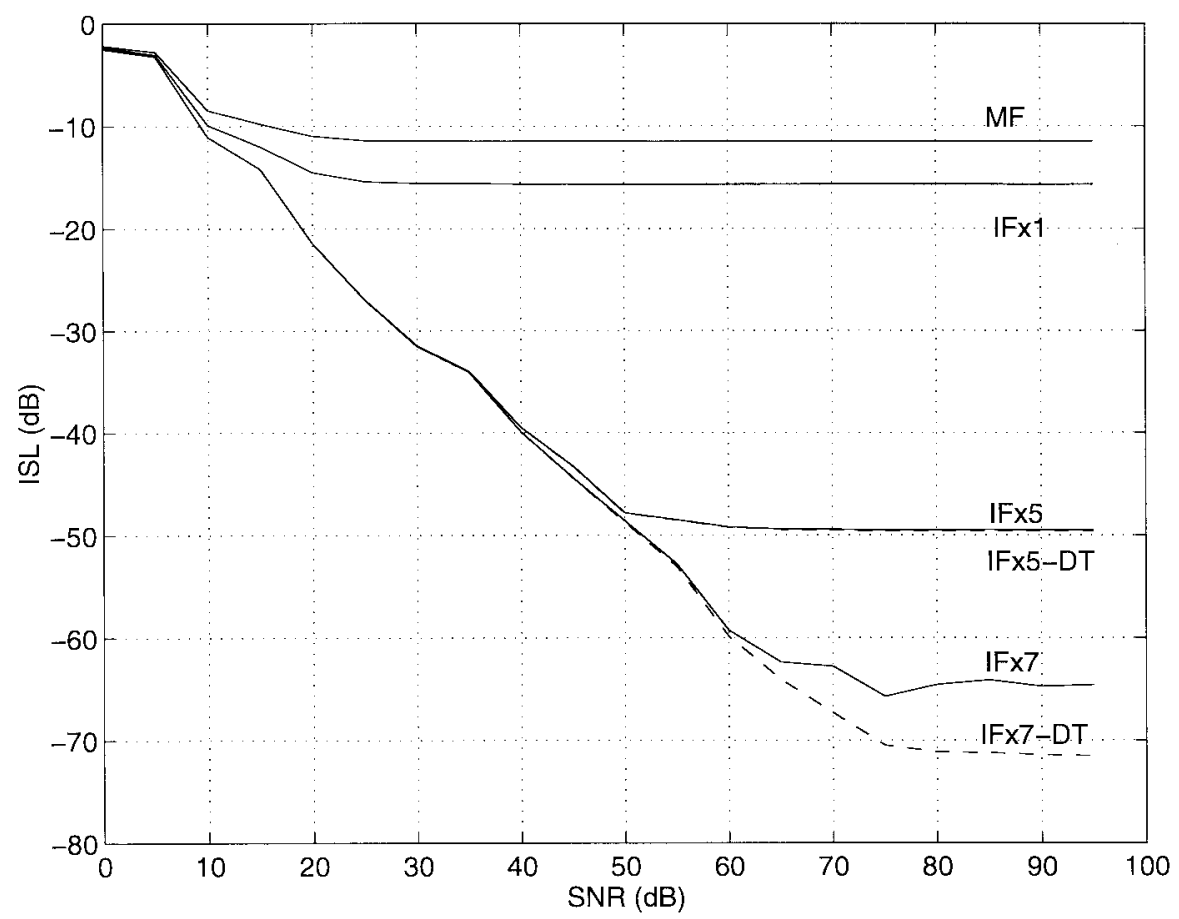

Fig. 10. ISL versus SNR for the B-13 waveform using inverse and matched filters for compression.

of the compression filter on the received signal. The SNR imposed limitation on the ISL is evident in Fig. 10 where the ISL is plotted as a function of SNR for a $100 \mathrm{~dB}$ fluctuating spike target with zero mean velocity and spectrum-width of 1 $\mathrm{m} / \mathrm{s}$. The solid lines in Fig. 10 represent the outputs of various filters and the dashed lines correspond to their Doppler tolerant counterparts. Examining the output of say the $\mathbf{I F} \times \mathbf{5}$ filter, we see that at SNR $=20 \mathrm{~dB}$, the ISL $\sim-20 \mathrm{~dB}$ and at SNR $=30$
$\mathrm{dB}$, the ISL $\sim-30 \mathrm{~dB}$, and so on until SNR $>50 \mathrm{~dB}$ where the ISL levels off at its minimum value of $-50 \mathrm{~dB}$. We therefore must be wary about arbitrarily extending filter lengths to get improved sidelobe suppression performance and recognize the role played by the SNR as a limiting factor in the sidelobe suppression performance of any filter.

3) Reflectivity and Velocity Step-Function Profiles: To evaluate the effects of gradients, an input profile consisting of 


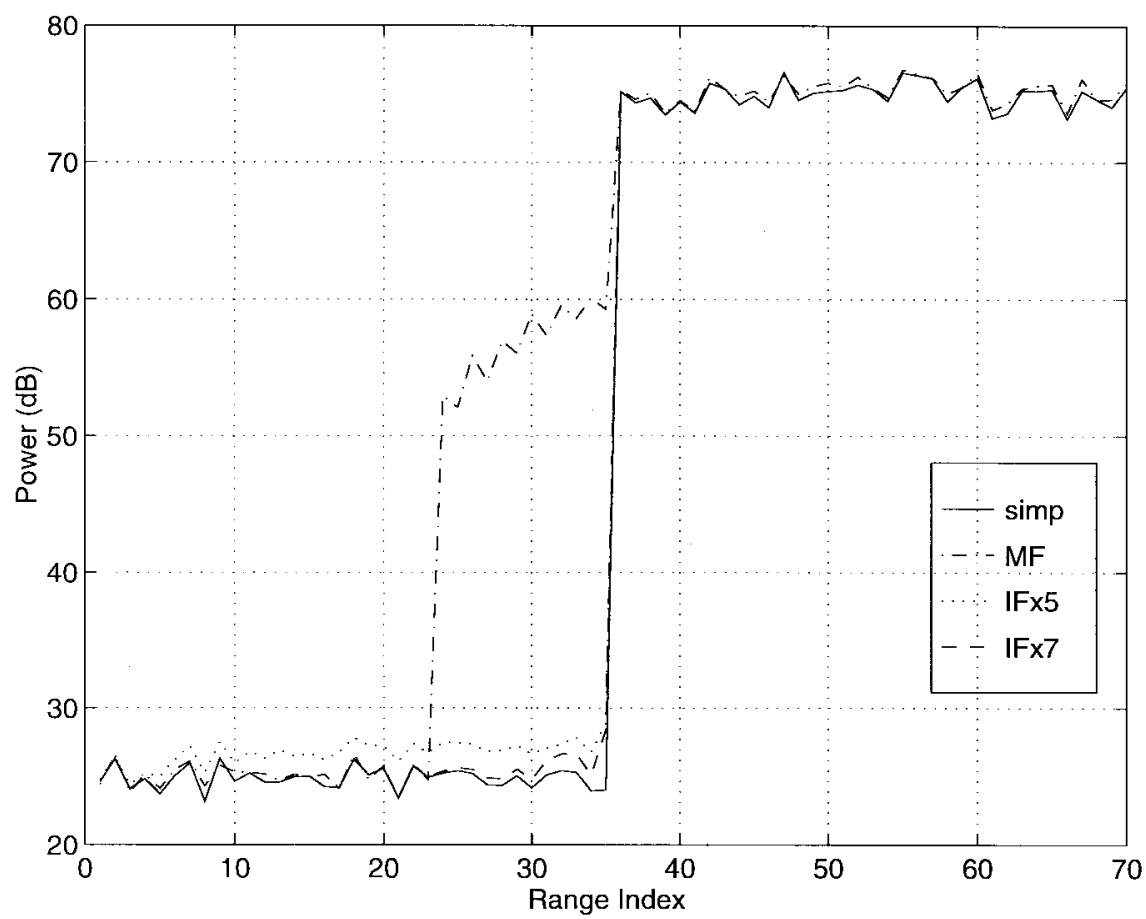

(a)

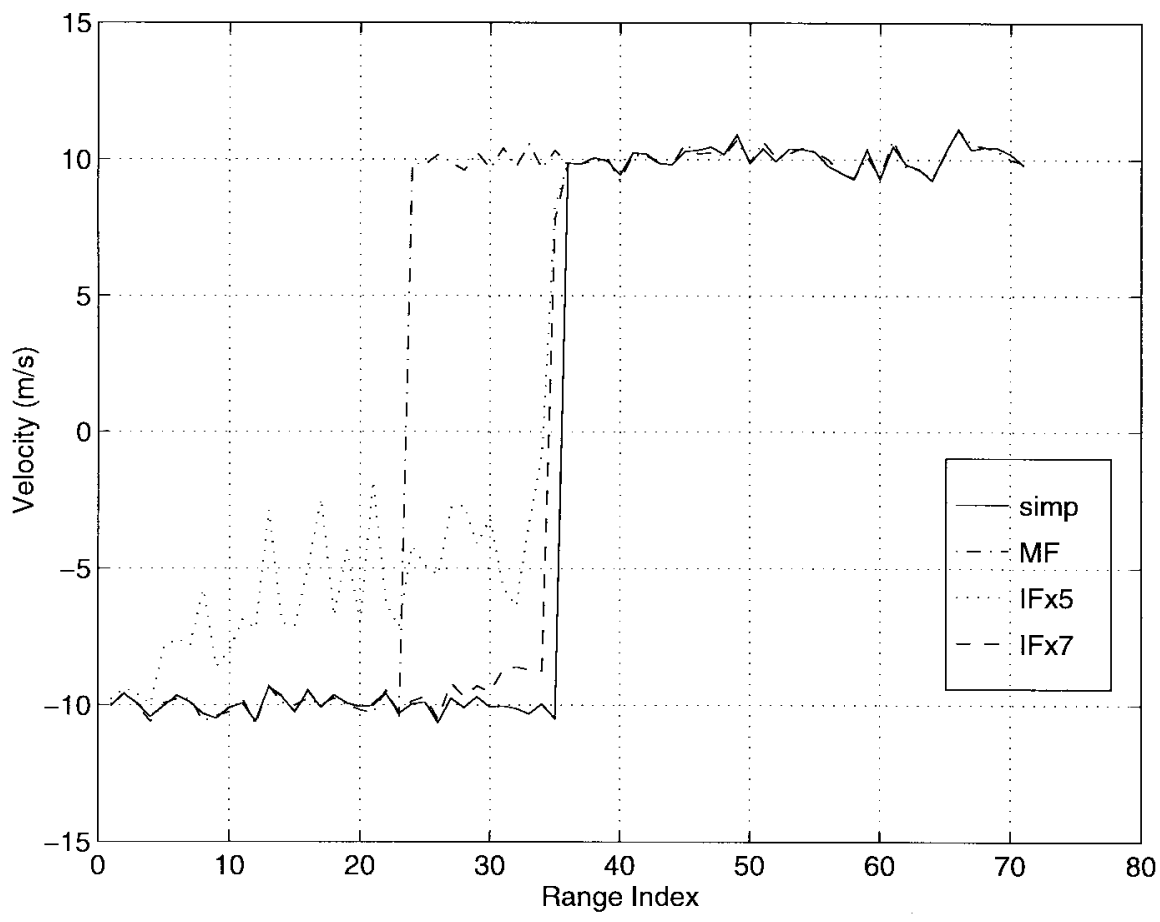

(b)

Fig. 11. Range profiles of (a) reflected power and (b) Doppler velocity. The input profile to the simulation has a 50-dB reflectivity step and a $20-\mathrm{m} / \mathrm{s}$ velocity step.

gradients in both reflectivity and velocity was used. Fig. 11 shows the range profiles of (a) the reflected power and (b) Doppler velocity at the outputs of the simple pulse $\mathbf{M F}, \mathbf{I F} \times \mathbf{5}$, and $\mathbf{I F} \times \mathbf{7}$. The reflectivity values on either side of the gradient differ by $50 \mathrm{~dB}$ and the velocity jumps from $-10 \mathrm{~m} / \mathrm{s}$ to +10 $\mathrm{m} / \mathrm{s}$. The SNR at all ranges was set at $60 \mathrm{~dB}$ with $\sigma_{v}$ at 2.5 $\mathrm{m} / \mathrm{s}$. It can be seen that the performance of $\mathbf{M F}$ in estimating both reflectivity and velocity is inferior to the inverse filters. The $\mathbf{I F} \times \mathbf{5}$ (best ISL $\sim-48 \mathrm{~dB}$ ) estimates of reflectivity at range bins just before the gradient are biased toward the higher reflectivity values past the gradient $(\sim 2-3 \mathrm{~dB}$ difference compared to simple pulse output) which is responsible for biasing the Doppler velocity estimates at these bins toward the velocities of the higher reflectivity targets. The $\mathbf{I F} \times \mathbf{7}$ 
(best ISL $\sim-70 \mathrm{~dB}$ ) does a good job of estimating both the reflected power and Doppler velocity. The best performance was obtained from the $\mathbf{I F} \times \mathbf{7}$-DT and the reflectivity and velocity profiles at the filter output very closely followed the simple pulse profiles (not shown in figure for clarity).

4) Input Profiles from Weather Data: The analysis of the performance of matched and inverse filters thus far was done using synthetic profiles for reflectivity, velocity, SNR, etc. These profiles illustrate the behavior of matched and inverse filters in extreme conditions and provide a worst-case analysis. When observing distributed weather targets, however, we say that any waveform-filter combination is suitable if it yields an ISL comparable to the nonmainlobe power from the twoway antenna pattern. In order to test this requirement, real weather data collected using the CSU-CHILL multiparameter weather radar were used to generate input range profiles for reflectivity $Z$, mean velocity $\bar{v}$, SNR, differential reflectivity $Z_{\mathrm{DR}}$, copolar correlation coefficient at lag $0 \rho_{\mathrm{HV}}(0)$, and differential phase-shift $\phi_{\mathrm{DP}}$ (see review article by Bringi $e t$ al. [21] for a description of these parameters). The spectrum width $\sigma_{v}$ was set at $2.5 \mathrm{~m} / \mathrm{s}$ at all ranges to better observe the degradation in sidelobe suppression performance due to factors other than the spectrum width. The data was collected on June 7, 1995, while observing an intense thunderstorm producing both rain and hail. The two main cores of precipitation, located at approximately 41 and $47 \mathrm{~km}$, show high reflectivities $(50-65 \mathrm{~dB})$. The $Z_{\mathrm{DR}}$ values at both cores are close to zero and the corresponding $K_{\mathrm{DP}}$ values are fairly large $\left(\sim 4^{\circ} / \mathrm{km}\right.$ corresponding to rain-rate of about $\left.130 \mathrm{~mm} / \mathrm{h}\right)$ indicating possible rain-hail mixture. The input reflectivity profile exhibits steep reflectivity gradients $(15-25 \mathrm{~dB} / \mathrm{km})$ and is a good candidate for sidelobe suppression evaluation. Fig. 12(a)-(f) shows range profiles of $Z, \bar{v}, \sigma_{v}, Z_{\mathrm{DR}}, \rho_{\mathrm{HV}}(0)$, and $\phi_{\mathrm{DP}}$. The solid line shows the values obtained from simple pulse simulation. The different dashed patterns represent the outputs of $\mathbf{M F}, \mathbf{I F} \times \mathbf{1}$, and $\mathbf{I F} \times \mathbf{5}$ for the coded pulse. It is evident from the figure that $\mathbf{M F}$ and $\mathbf{I F} \times \mathbf{1}$ estimates of various parameters have unacceptable errors near the gradient regions. The $\mathbf{I F} \times \mathbf{5}$ filter, however, matches the simple pulse response and performs well in these regions. Other filter outputs are not shown in figure for the sake of clarity.

In order to quantify the performance of various filters, we define the mean deviation of variable $x$ as $M D(x)=$ $E\left[a b s\left(x_{\mathrm{pc}}-x_{s}\right)\right]$, where the expectation is carried out over all range bins. $x_{s}$ and $x_{\mathrm{pc}}$ refer to estimates of $x$ obtained from the output of simple pulse simulation and pulse compression simulation, respectively. Table I shows the mean deviation of various parameters at the output of the matched and inverse filters. It can be seen from Table I that with increasing filter length, the accuracy of the estimates gets better. Depending on the required accuracy of the multiparameter estimates, it appears that for reflectivity gradients encountered in typical weather situations, the $\mathbf{I F} \times \mathbf{5}$ or the $\mathbf{I F} \times \mathbf{7}$ (ISL $<-45 \mathrm{~dB}$ ) provide sidelobe suppression adequate to represent the actual reflectivity structure. Also, the improvement obtained from the Doppler-tolerant implementation is not very significant in this case. This could be due to the fact that the input velocity profile to the simulation is confined to the interval $(-6,10)$
TABLE I

Mean Deviation of Various Parameters at the Output of Matched and Inverse Filters for B-13 Phase Code

\begin{tabular}{c|c|c|c|c|c|c}
\hline & MF & IFx1 & IFx5 & IFx5-DT & IFx7 & IFx7-DT \\
\hline$Z(d B)$ & 2.6268 & 1.9658 & 0.1621 & 0.1606 & 0.0731 & 0.0706 \\
\hline $\bar{v}(\mathrm{~m} / \mathrm{s})$ & 1.1586 & 0.9729 & 0.2803 & 0.2779 & 0.1060 & 0.1034 \\
\hline$\sigma_{v}(\mathrm{~m} / \mathrm{s})$ & 0.3684 & 0.3429 & 0.1632 & 0.1640 & 0.0369 & 0.0372 \\
\hline$Z_{D R}(\mathrm{~dB})$ & 0.4128 & 0.3165 & 0.1102 & 0.1105 & 0.0867 & 0.0856 \\
\hline$\rho_{H V}(0)$ & 0.0442 & 0.0405 & 0.0120 & 0.0119 & 0.0084 & 0.0084 \\
\hline$\phi_{D P}(\mathrm{deg})$ & 4.2325 & 3.6317 & 1.7767 & 1.7749 & 1.3812 & 1.3655 \\
\hline
\end{tabular}

$\mathrm{m} / \mathrm{s}$ and the degradation in ISL of inverse filters at these velocities is not significant for the reflectivity gradients under consideration.

\section{DATA EVAluation For FAST-SCAN APPLICATIONS}

The CSU-CHILL radar transmits $1 \mathrm{~mW}$ peak power and it is not easy to implement large bandwidth waveforms with such high-power transmitters. However, a phase-coded pulse compression scheme has been implemented through the use of a phase modulator interfaced with the RF synthesis circuit in the transmitter. Operating at a frequency of $60 \mathrm{MHz}$, the modulator is capable of shifting the phase in $90^{\circ}$ increments every $200 \mathrm{~ns}$. This phase shifted $60 \mathrm{MHz}$ signal is mixed with the $2.785 \mathrm{GHz}$ signal and the difference signal is sent to an amplifier and fast pulse modulator to produce a $1 \mu$ s pulse. There are two such units in order to facilitate complementary coding. A wideband intermediate power amplifier has been acquired to accommodate the higher bandwidth of the signal. Both biphase and quadriphase codes of length 5 were used to modulate the phase of the $1-\mu \mathrm{s}$-long transmit pulse. On reception, the received signal was sampled at the inverse bandwidth rate $\left(T_{s}=1 / 5 \mathrm{MHz}=200 \mathrm{~ns}\right)$ corresponding to $30-\mathrm{m}$ range spacing. The received I/Q time series data was processed off line to test the various pulse compression algorithms.

\section{A. Data Analysis}

On August 30, 1995, time-series data was collected over a storm to the Northwest of the CSU-CHILL radar. The antenna position was fixed pointing at the core of the storm. As before, the transmit polarization was set at horizontal $(\mathrm{H})$, and the received signal was sampled every $200 \mathrm{~ns}$ (corresponding to $30 \mathrm{~m}$ in range). Time series data was collected with the transmit pulse-width $(\tau)$ set at $1 \mu \mathrm{s}$, with and without phase coding. Various 5-bit phase codes, including Barker and complementary codes, were used. Fig. 13 shows the standard deviation in estimates of reflectivity factor $10 \log \bar{P}$ as a function of number of samples used to form the mean estimate, at a fixed resolution cell. The power values from five adjacent range bins, i.e., reference bin and the two bins on either side of the reference bin, were averaged and therefore, the net range sampling interval was increased to $150 \mathrm{~m}$ (corresponding to the range resolution of the uncoded pulse). Note that the lowered 


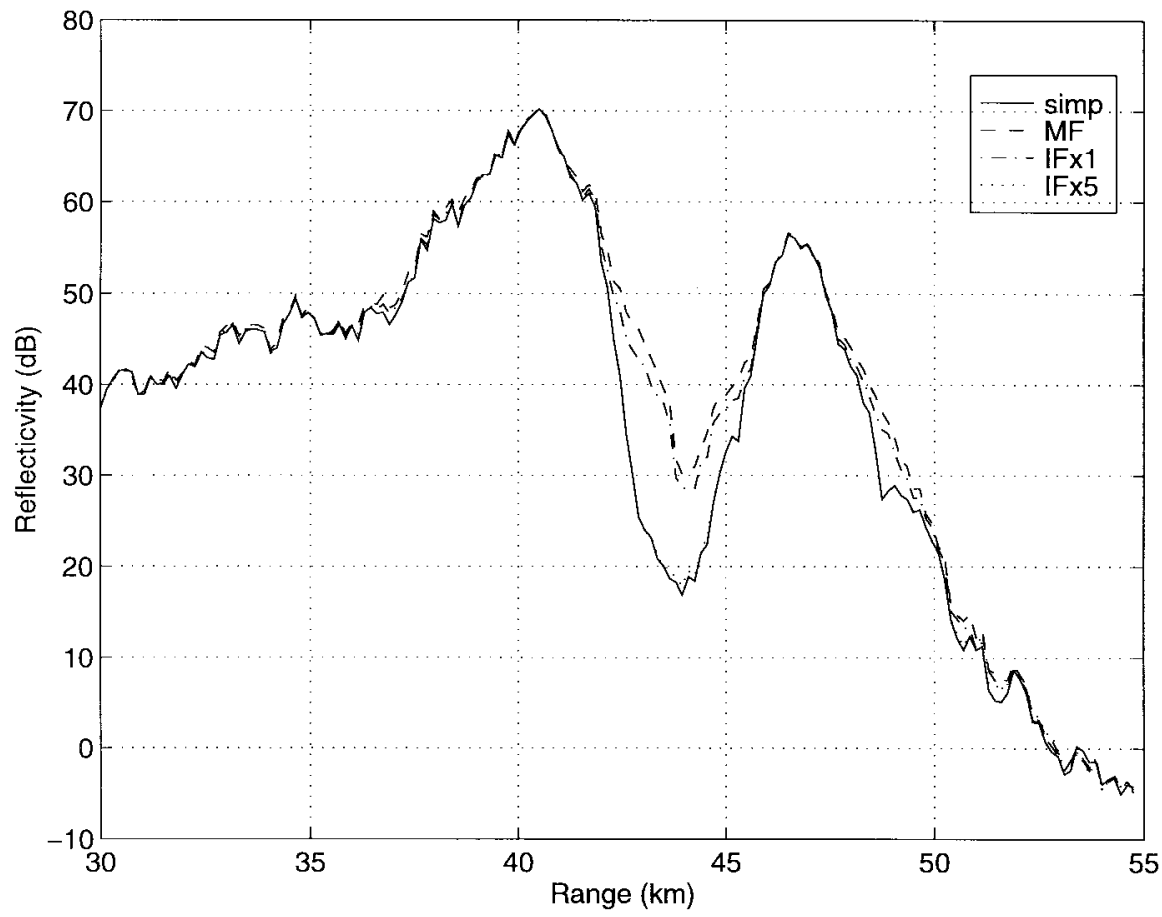

(a)

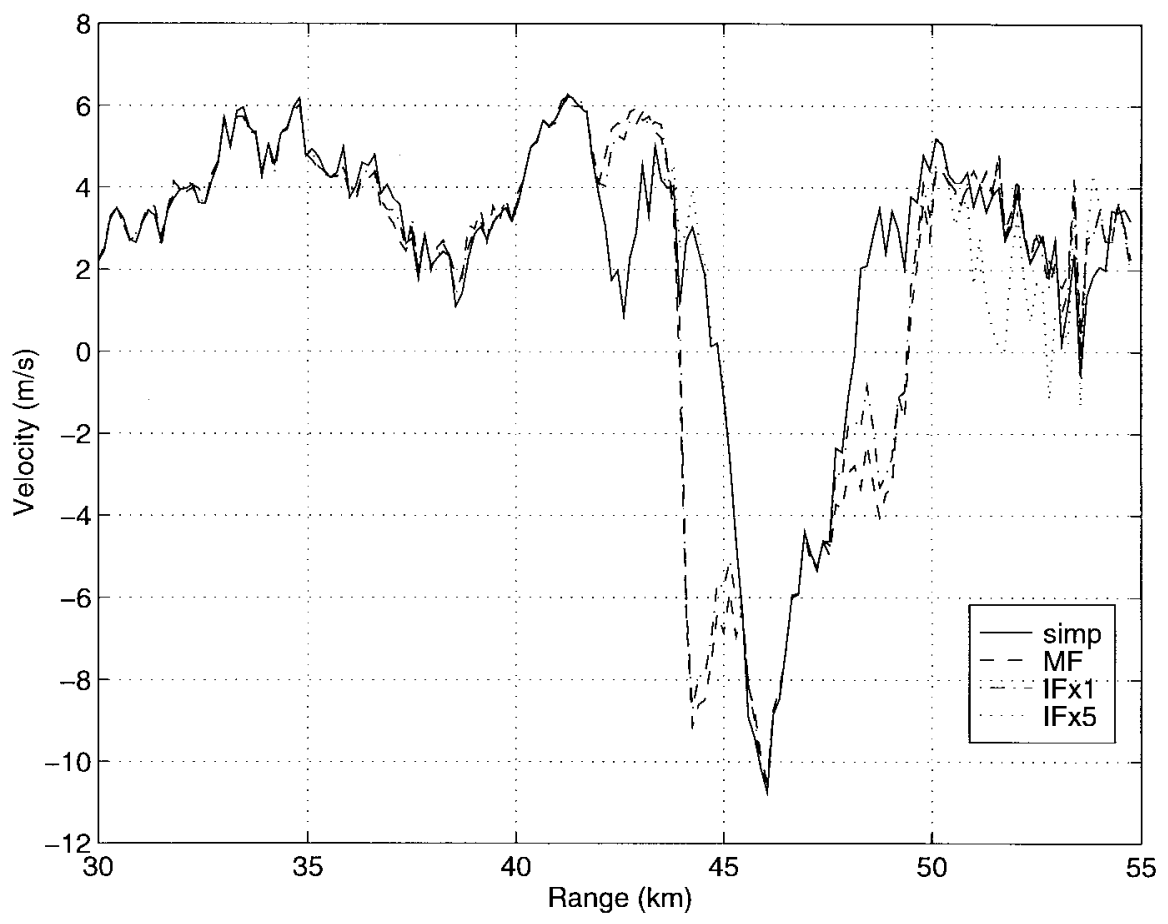

(b)

Fig. 12. Range profiles of (a) $Z(\mathrm{~dB})$ and $(\mathrm{b}) \bar{v}(\mathrm{~m} / \mathrm{s})$. The different curves show the profile from the simple pulse and the outputs of $\mathbf{M F}$, IF $\times \mathbf{1}$, and $\mathbf{I F} \times \mathbf{5}$ for the $\mathrm{B}-13$ coded pulse.

values for standard deviation (by approximately the expected $\sqrt{5}$ ) for the pulse compression data is due to the fact that more independent samples in range are available for averaging than for the uncoded pulse.

Also, note that the various pulse compression schemes, the Barker coded, $\mathbf{I F} \times \mathbf{5}$ processed output yields the lowest stan- dard deviation followed by the complementary coding scheme and the Barker code, MF processed outputs, respectively.

\section{SUMmary AND CONCLUSIONS}

A new simulation procedure has been developed which accurately models the joint distribution properties of fluctuating 


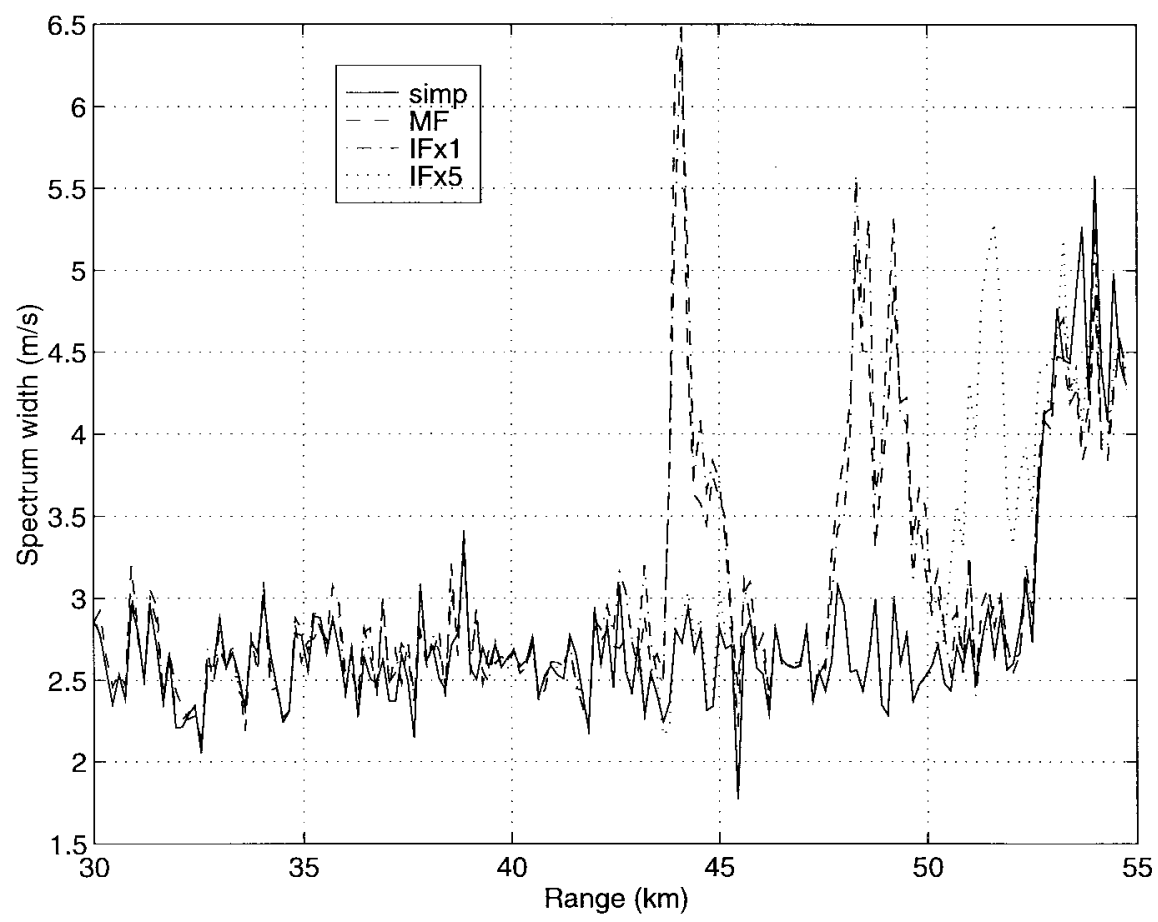

(c)

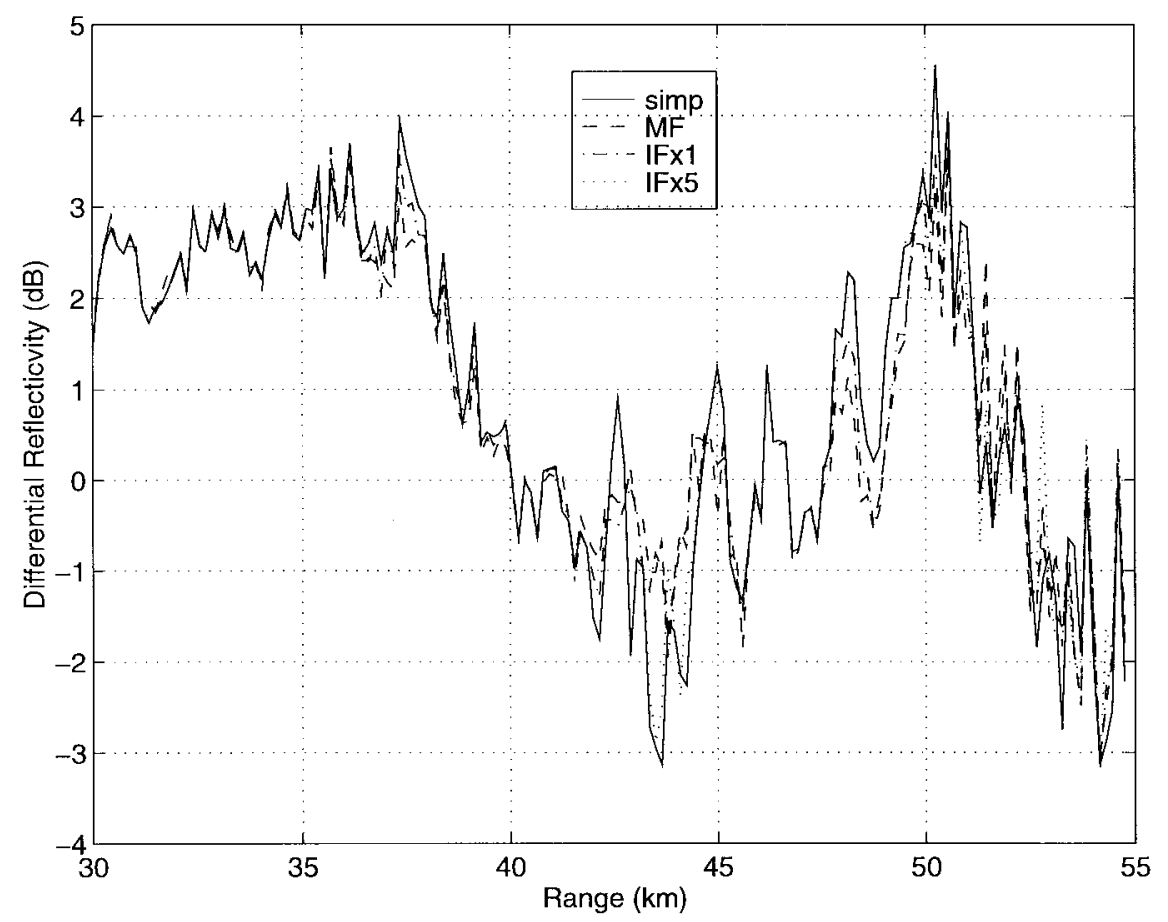

(d)

Fig. 12. (Continued.) Range profiles of (c) $\sigma_{v}(\mathrm{~m} / \mathrm{s})$ and (d) $Z_{\mathrm{DR}}(\mathrm{dB})$ The different curves show the profile from the simple pulse and the outputs of $\mathbf{M F}, \mathbf{I F} \times \mathbf{1}$, and $\mathbf{I F} \times \mathbf{5}$ for the B-13 coded pulse.

weather radar echoes with pulse compression. This procedure accounts for the reshuffling of scatterers within the resolution volume during the pulse propagation time which is important, especially for longer pulse lengths. The procedure generates time-records for both single and dual polarization operations. The performance of various compression and sidelobe sup- pression filters were evaluated using simulation output and pulse compression data for both point targets and time-varying distributed targets. The criteria used in evaluation were

- integrated sidelobe level (ISL);

- Doppler sensitivity over the velocity interval commonly encountered with meteorological targets, i.e., $[0,50] \mathrm{m} / \mathrm{s}$; 


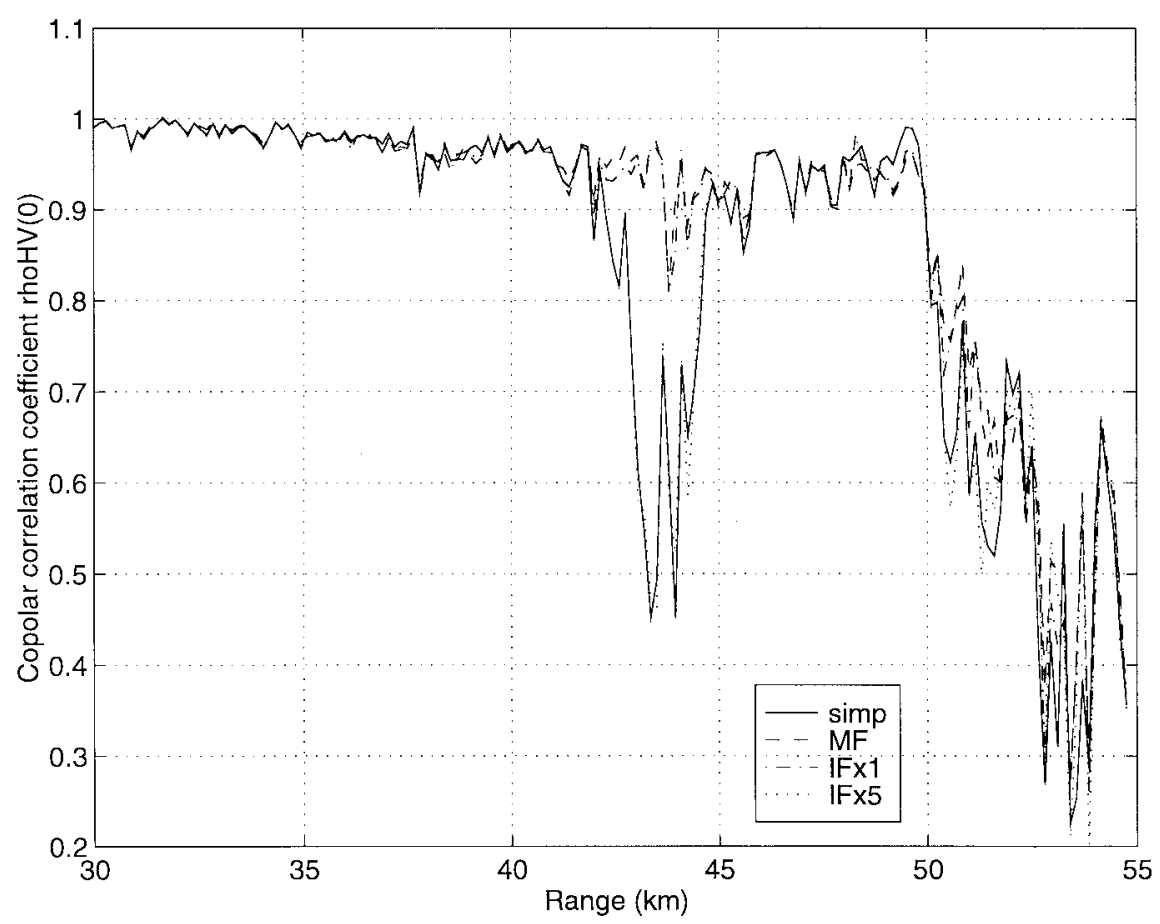

(e)

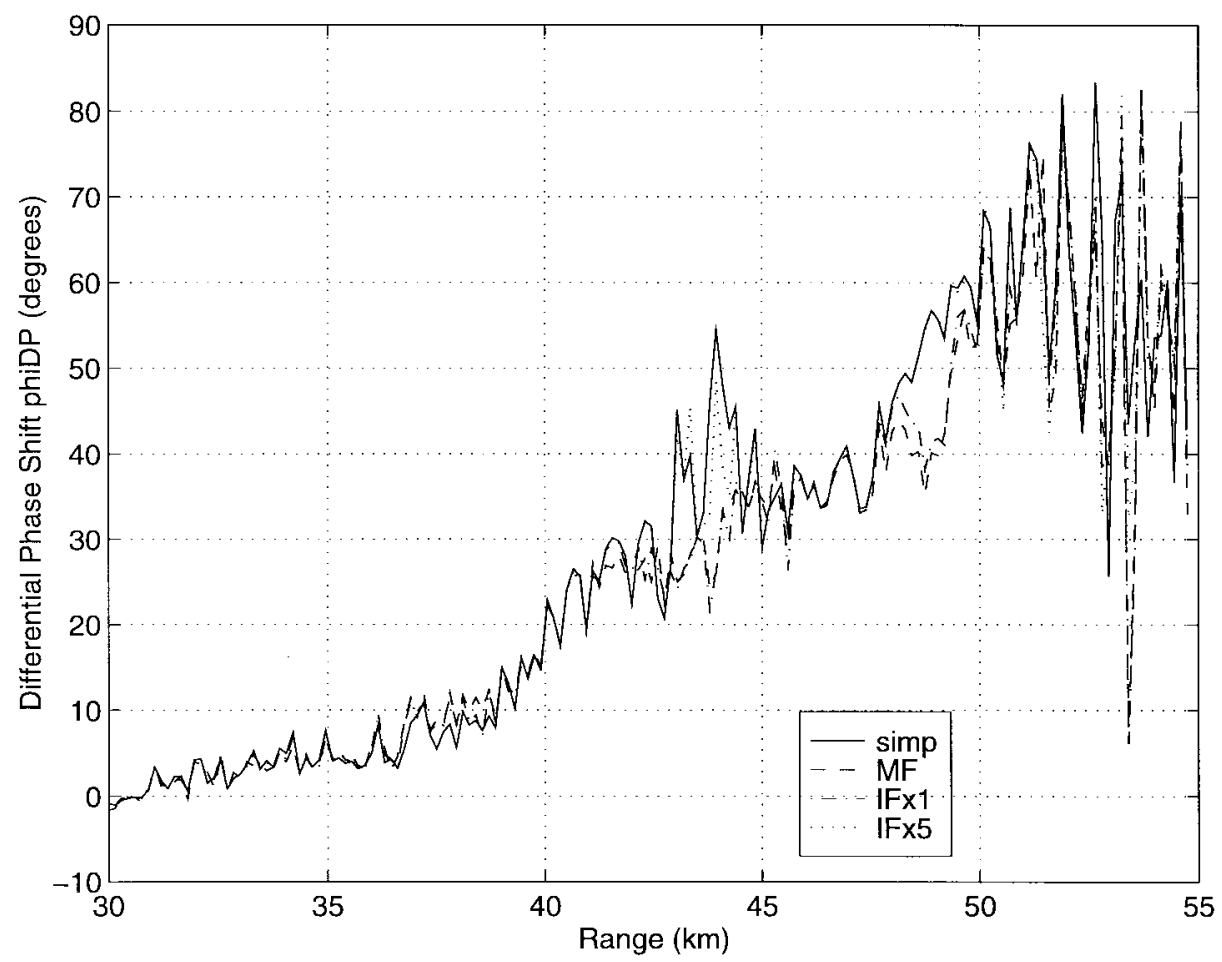

(f)

Fig. 12. (Continued.) Range profiles of (e) $\left|\rho_{\mathrm{HV}}(0)\right|$ and (f) $\phi_{\mathrm{DP}}\left({ }^{\circ}\right)$. The different curves show the profile from the simple pulse and the outputs of $\mathbf{M F}, \mathbf{I F} \times \mathbf{1}$, and $\mathbf{I F} \times \mathbf{5}$ for the B-13 coded pulse.

- comparison with output from simple pulse waveform of equal bandwidth.

Our analysis shows that the level of suppression offered by the matched filter is not sufficient in cases with strong gradients in reflectivity. Inverse filters, designed to minimize the integrated sidelobe level, seem to perform more than adequately for commonly expected weather gradients, especially at longer filter lengths (five or more times longer than waveform). The same performance carries over to dual polarization implementations, i.e., good estimates of polarimetric variables can be obtained.

It is shown that the sidelobe suppression performance of inverse filters degrades considerably with increasing mean Doppler velocities of the weather targets. Evaluation based 


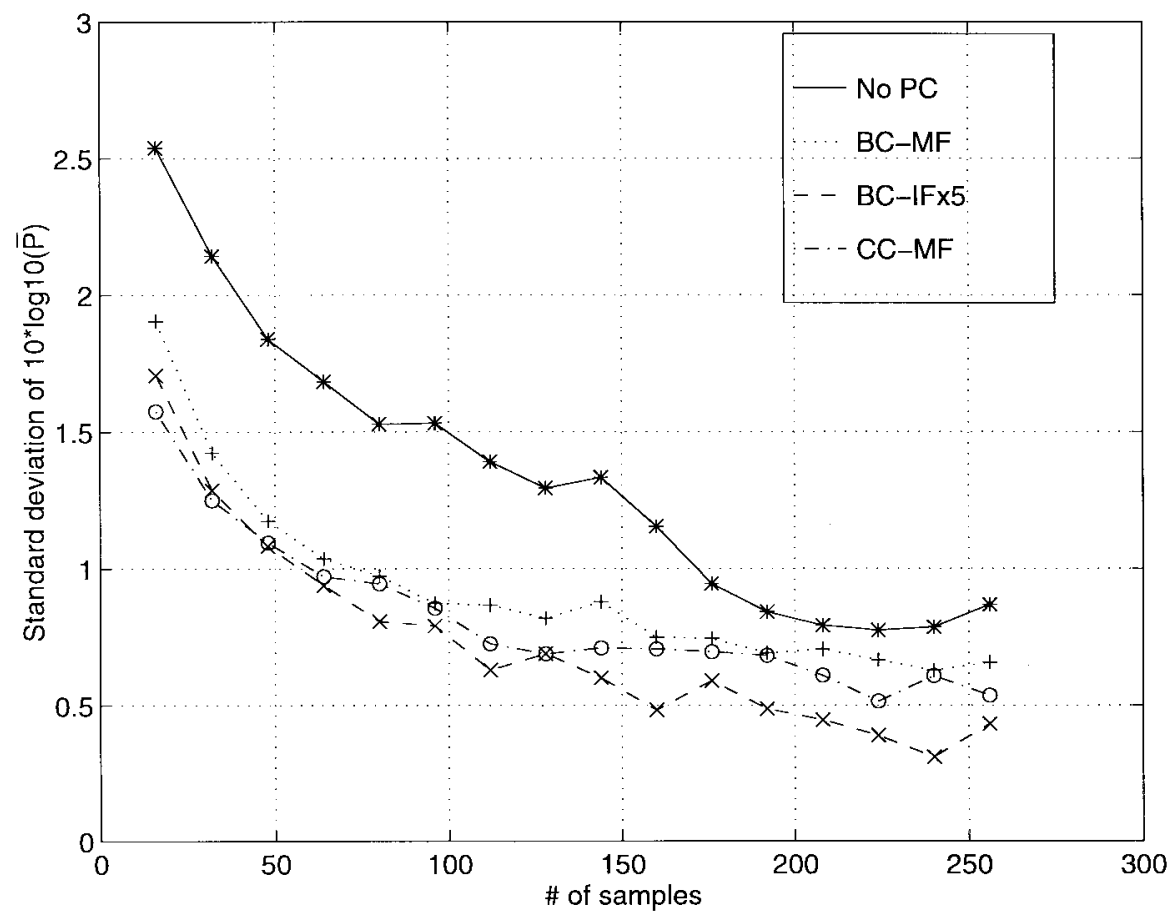

Fig. 13. Standard deviation of $10 \log _{10} \bar{P}$ versus number of samples used in estimate. Various curves represent output from uncoded pulse (solid), 5-bit Barker coded pulse with $\mathbf{M F}>$ processing (dotted), 5-bit Barker coded pulse with $\mathbf{I F} \times \mathbf{5}$ processing (dashed), and 5-bit complementary coding (dash-dot).

on simulation using steep reflectivity gradients show that the Doppler tolerant implementation helps maintain low sidelobe levels even at higher Doppler velocities. This scheme, however, is effective only when the full Doppler spectrum of the radar returns are confined to the unambiguous Nyquist interval. With increasing spectrum width, the range of velocities that can be processed effectively narrows down significantly.

For low SNR, the performance of all range sidelobe suppression filters were shown to be limited by the SNR. In these cases, arbitrarily increasing filter length does not necessarily yield lower sidelobe levels. Thus, the SNR imposed limit on the ISL can cause significant sidelobe contamination in measurements made while observing weak echo regions using pulse compression weather radars. This analysis also shows that for high SNR cases where noise does not limit the ISL, the longer filters do generally reduce the ISL.

One of the main applications of high-resolution measurements is to improve accuracy in estimates of radar parameters such as $Z, Z_{\mathrm{DR}}$, etc., through range averaging. For example, using a 5-bit Barker code and averaging over five adjacent range bins, the standard deviation in reflectivity estimates was reduced by a factor of about $\sqrt{5}$. With longer codes, better improvement can be achieved. These improvements in accuracy of radar measurements are not at the expense of increased dwell time. Currently, weather radars operated by the National Weather Service (NEXRAD) use scan-rates of about $18-20 \%$ in precipitation mode corresponding to integration of 50 samples. However for multiparameter radars, due to accuracy requirements in estimates polarimetric variables, integration over 64 sample-pairs (i.e., 128 samples) is usually considered acceptable. This necessitates slower antenna scan rates $(6-8 \%$ s). Using pulse compression techniques with multiparameter radars, accuracies equal to or better than those currently available can be achieved at NEXRAD-like scanrates.

\section{ACKNOWLEDGMENT}

The authors acknowledge very helpful discussions with Dr. V. N. Bringi at Colorado State University and the CSU-CHILL radar staff with their assistance in obtaining pulse compression data.

\section{REFERENCES}

[1] C. E. Cook and M. Bernfeld, Radar Signals: An Introduction to Theory and Application. New York: Academic, 1967.

[2] A. W. Rihaczek, Principles of High Resolution Radar. New York: McGraw-Hill, 1969.

[3] R. W. Fetter, "Radar weather performance enhanced by pulse compression," in Preprints, 14th AMS Conf. Radar Meteorol., Tucson, AZ, Nov. 1970, pp. 413-418.

[4] R. W. Gray and D. T. Farley, "Theory of incoherent-scatter measurements using compressed pulses," Radio Sci., vol. 8, no. 2, pp. 123-131, Feb. 1973.

[5] R. J. Keeler and R. E. Passarelli, "Signal processing for atmospheric radars," in Proc. Radar Meteorol., pp. 199-229, 1990.

[6] R. J. Keeler and C. A. Hwang, "Pulse compression for weather radar," in Proc. IEEE Int. Radar Conf., May 1995, pp. 529-535.

[7] J. G. Weiler and F. Ohora, "Stormfinder 2000: A solid-state S-band weather radar system characteristics," in Preprints, 27th AMS Conf. Radar Meteorol., Vail, CO, Oct. 1995, pp. 720-722.

[8] M. N. Cohen and F. E. Nathanson, "Phase-coding techniques," Radar Design Principles. New York: McGraw-Hill, 1990, ch. 12.

[9] M. I. Skolnik, Introduction to Radar Systems. New York: McGrawHill, 1980, pp. 371-373.

[10] P. M. Woodward, Probability and Information Theory, With Applications to Radar. New York: McGraw-Hill, , 1953.

[11] J. S. Marshall and W. Hitschfeld, "The interpretation of the fluctuating echo from randomly distributed scatterers, Part 1," Can. J. Phys., vol. 31, pp. 962-994, 1953.

[12] R. J. Doviak and D. S. Zrnić, Doppler Radar and Weather Observations. San Diego, CA: Academic, 1984. 
[13] V. Chandrasekar, V. N. Bringi, and P. J. Brockwell, "Statistical properties of dual-polarized radar signals," in Proc. 23rd AMS Conf. Radar Meteorol., Snowmass, CO, Sept. 1986, pp. 193-196.

[14] N. J. Bucci and H. Urkowitz, "Testing of doppler tolerant range sidelobe suppression in pulse compression meteorological radar," in Proc. IEEE Nat. Radar Conf., Boston, MA, Apr. 1993, pp. 206-211.

[15] M. H. Ackroyd and F. Ghani, "Optimum mismatched filters for sidelobe suppression,” IEEE Trans. Aerosp. Electron. Syst., vol. AES-9, pp. 214-218, Mar. 1973.

[16] N. J. Bucci, "Doppler tolerant range sidelobe suppression for pulse compression radars. Tech. Rep. MTMR-91-TR-001, GE Aerospace, Moorestown, NJ, 1991.

[17] S. Trietel and E. A. Robinson, "The design of high resolution digital filters," IEEE Trans. Geosci. Electron., vol. GE-4, pp. 25-38, June 1966.

[18] H. Urkowitz and N. J. Bucci, "Doppler tolerant range sidelobe suppression for meteorological radar with pulse compression," in Proc. IGARSS, Houston, TX, May 1992, vol. 1, pp. 206-208.

[19] A. Mudukutore, V. Chandrasekar, and R. J. Keeler, "Range sidelobe suppression for weather radars with pulse compression: Simulation and evaluation," in Preprints, 27th AMS Conf. Radar Meteorol., Vail, CO, Oct. 1995, AMS, pp. 763-766.

[20] A. Mudukutore, V. Chandrasekar, and R. J. Keeler, "Simulation and analysis of pulse compression for weather radars," in Proc. IGARSS, Firenze, Italy, July 1995

[21] N. N. Bringi, V. Chandrasekar, P. Meischner, J. Hubbert and Y. Golestani, "Polarimetric radar signatures of precipitation at S- and Cbands" Proc. Inst. Elect. Eng., pt. F. vol. 138, no. 2, pp. 109-119, 1991.

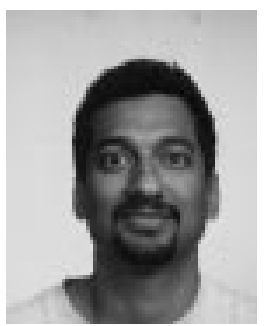

Ashok S. Mudukutore received the B.E. degree in electronics and communications from the University of Mysore, Mysore, India in 1988 and the M.S. and $\mathrm{Ph} . \mathrm{D}$. degrees in electrical engineering from Colorado State University (CSU), Fort Collins, in 1992 and 1996, respectively.

He was a Research Assistant at CSU while pursuing the Ph.D. degree. He is presently a Research Engineer at the Center for Aerospace Technology, Research Triangle Institute, Hampton, VA.
V. Chandrasekar ( $\mathrm{S}^{\prime} 83-\mathrm{M}^{\prime} 87$ ), photograph and biography not available at the time of publication.

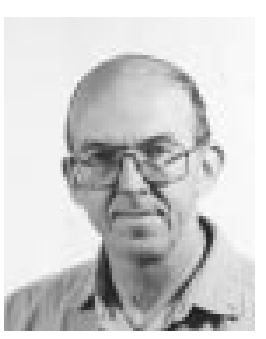

R. Jeffrey Keeler (S'63-M'66) received the B.S.E.E. degree from the Rose-Hulman Institute of Technology, Terre Haute, IN, the M.S.E.E. from Stanford University, Stanford, CA, and the Ph.D. degree, in 1979, in electrical engineering from the University of Colorado, Boulder

$\mathrm{He}$ has previously worked at the NOAA/ERL Wave Propagation Laboratory, Boulder, and Bell Laboratories, Holmdel, NJ. He is presently a Research Engineer and Manager of the Remote Sensing Facility, National Center for Atmospheric Research, Boulder, where he coordinates remote sensor development and deployment activities including UHF, microwave, and optical remote sensors. $\mathrm{He}$ is an Adjunct Professor at Colorado State University, Fort Collins, where he teaches courses on meteorological radar technology and applications. His current research interest center on advanced weather radar system design, which rely on phased array antennas and pulse compression waveform design and processing techniques, as well as optical remote sensing techniques. 